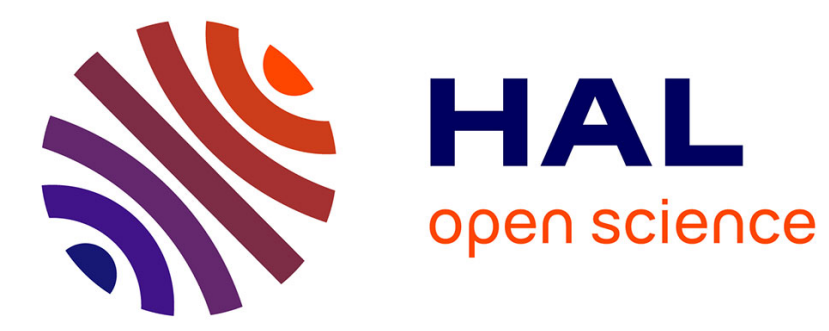

\title{
Coherent-vortex dynamics in large-eddy simulations of turbulence
}

Marcel Lesieur, Patrick Bégou, Emmanuel Briand, Alexandra Danet, Franck Delcayre, Jean-Luc Aider

\section{- To cite this version:}

Marcel Lesieur, Patrick Bégou, Emmanuel Briand, Alexandra Danet, Franck Delcayre, et al.. Coherent-vortex dynamics in large-eddy simulations of turbulence. Journal of Turbulence, 2003, 4, 10.1088/1468-5248/4/1/016 . hal-00261421

\section{HAL Id: hal-00261421 \\ https://hal.science/hal-00261421}

Submitted on 19 Mar 2020

HAL is a multi-disciplinary open access archive for the deposit and dissemination of scientific research documents, whether they are published or not. The documents may come from teaching and research institutions in France or abroad, or from public or private research centers.
L'archive ouverte pluridisciplinaire HAL, est destinée au dépôt et à la diffusion de documents scientifiques de niveau recherche, publiés ou non, émanant des établissements d'enseignement et de recherche français ou étrangers, des laboratoires publics ou privés. 


\title{
Coherent-vortex dynamics in large-eddy simulations of turbulence
}

\author{
M Lesieur ${ }^{1}$, P Begou ${ }^{1}$, E Briand ${ }^{1}$, A Danet ${ }^{1}$, \\ F Delcayre ${ }^{1}$ and J L Aider ${ }^{2}$ \\ ${ }^{1}$ LEGI, BP 53, 38041 Grenoble Cedex 9, France \\ ${ }^{2}$ PSA Peugeot-Citroën, DRIA/SARA/PVMO, 78943 Velizy-Villacoublay \\ Cedex, France \\ E-mail: Marcel.Lesieur@hmg.inpg.fr
}

\begin{abstract}
We present a review of coherent-vortex dynamics obtained thanks to large-eddy simulations (LES) associated with simple and effective vortexidentification and animation techniques. LES of a large class of constant-density or weakly compressible three-dimensional flows have been carried out. In isotropic turbulence, we present the formation and evolution of spaghetti-type vortices, seen thanks to $Q$, vorticity and pressure, together with the time evolution of the kinetic energy, enstrophy and skewness. In a spatially growing boundary layer on a flat plate, one observes during transition big $\Lambda$ vortices lying on the wall (with very well correlated oblique induced low- and high-speed streaks) shedding smaller hairpin vortices around their tips. In the developed boundary layer, we show animations of the purely longitudinal low- and high-speed streaks, as well as animations of low-pressure regions. In a backwards-facing step, we examine the influence of upstream conditions upon the flow structure, by comparing two inflow conditions: a white noise superposed on a mean velocity profile and a realistic turbulent boundary layer. The latter three-dimensionalizes the flow downstream of the step and reduces the reattachment length. In both cases big staggered arch vortices form, impinge the lower wall and are carried away downstream. In a two-dimensional (2D) square cavity, spanwisely oriented vortices are shed behind the upstream edge, and impinge the downstream edge, transforming into arch vortices very similar to the back-step case. These arch vortices are also found behind a $2 \mathrm{D}$ rectangular obstacle with wall effect. We discuss the relevance of the vortices found with respect to reality. All these eddies are very important in terms of drag and noise reduction in aerodynamics and aeroacoustics.
\end{abstract}

PACS numbers: 47.27.Eq, 47.27.Gs, 47.27.Nz, 47.32.Ff 


\section{Contents}

1 Introduction $\quad 2$

2 Decaying three-dimensional isotropic turbulence $\quad 2$

3 Boundary layer developing on a flat plate $\quad 8$

4 Backward-facing step $\quad 10$

5 Two-dimensional square cavity $r$

6 2D rectangular obstacle with wall effect 13

$\begin{array}{llr}7 & \text { Conclusion and perspectives } & 20\end{array}$

\section{Introduction}

This work is a review of recent large-eddy simulations (LES) of constant-density or weakly compressible turbulent flows carried out in Grenoble. Emphasis is put mainly on the dynamics of coherent vortices which are observed within these LES. It will be seen that the vortices found have a universal character, in the sense that the various types of structure encountered are not numerous.

The flows considered will be either of uniform density (decaying isotropic turbulence, incompressible backward-facing step), or weakly compressible (boundary layer developing on a flat plate, back-step, two-dimensional (2D) square cavity and 2D rectangular obstacle with wall effect).

In isotropic turbulence, thanks to LES one recovers the spaghetti-type vortices which were first found in the direct numerical simulations (DNS) of Siggia [1] (who called them bananas). The origin of these vortices as resulting from a Kelvin-Helmholtz type instability of vortex sheets formed during an initial stage of evolution is not obvious in our LES.

In detached flows, we see spiral Kelvin-Helmholtz vortices very clearly, which are realigned into big longitudinal vortices which travel with the flow. Here, LES are a good tool of study, since instabilities controlling these processes are of an inviscid type, and not very much affected by the subgrid eddy viscosity if the latter is not sensitive to the large-scale shears. This is the case of subgrid models chosen in the following simulations. In boundary layers, we find with LES thin quasi-longitudinal vortices, as in DNS and laboratory experiments.

\section{Decaying three-dimensional isotropic turbulence}

It is well known that coherent vortices exist in developed three-dimensional (3D) isotropic turbulence (both forced and freely decaying), in the form of thin randomly orientated tubes where vorticity has concentrated. Their length is approximately the turbulence integral scale. As already mentioned, they were first discovered in forced DNS (at low Reynolds number) by Siggia [1], and their existence was confirmed still with DNS at higher Reynolds number by She et al [2], Vincent and Ménéguzzi [3] and Jimenez and Wray [4]. Métais and Lesieur [5] found them in LES of decaying turbulence at zero molecular viscosity, and checked there was some correlation between high-vorticity tubes and low-pressure ones. Here, the pressure is a 'macropressure' $P$, which is the filtered pressure corrected to account for the trace of the subgrid-stress tensor, and is eliminated from the filtered momentum equation with the aid of the filtered 


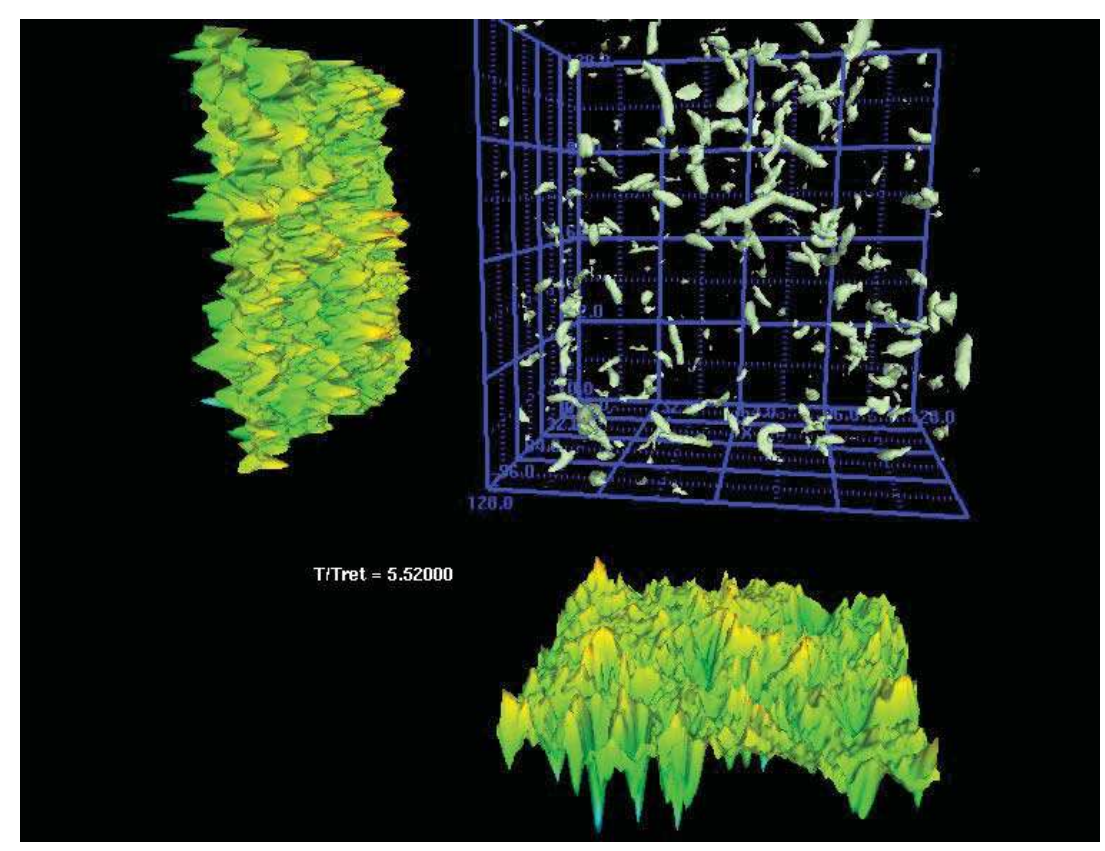

Figure 1. Animation of low-macro-pressure isosurfaces from $t=0$ to 15 initial large-eddy turnover times, $k_{i}=4,128^{3}$ modes.

continuity equation. Experimentally, similar vortices were found by Cadot et al [7]. A very simple argument to explain the low-pressure/high-vorticity correlation (see [6] and [7]) is that, in a frame linked to a fluid parcel assumed to wind around a coherent vortex, the parcel is approximately in balance between centrifugal and pressure-gradient forces, and therefore the vortex centre will be a pressure trough. Let us also mention that Robinson [8] used pressure to characterize vortices in boundary layers.

Dubief and Delcayre [9] compared in DNS of decaying isotropic turbulence various criteria to identify coherent vortices, including high vorticity modulus and low pressure, and also found a good correlation, although the low-pressure tubes are fatter and involve larger scales than their vorticity-based counterparts. But, up to now, no animation of these vortices has been published in a regular journal.

In [10], various LES of decaying 3D incompressible isotropic turbulence using the spectraldynamic model [11] starting with an initial Gaussian velocity field (of kinetic-energy spectrum decreasing exponentially at high $k$ ) and without molecular viscosity were developed, with emphasis put on the large-scale and infrared kinetic-energy and pressure statistics. For this purpose, the initial kinetic-energy infrared spectral exponent $s_{0}$, such that the initial energy spectrum $E(k, 0) \propto k^{s_{0}}$ as $k \rightarrow 0$, was varied, and the energy peak was put close to the cut-off wavenumber $k_{C}$. Confirmation of the infrared $k^{4}$ energetic spectral backscatter was provided, as well as a time-decaying $k^{2}$ infrared pressure spectrum. In the present work, we rather focus on coherent-vortex dynamics, studied in a LES using the same subgrid model. We take $s_{0}=4$. We will start with simulations involving $128^{3}$ collocation points, and initial spectral peak of $k_{i}=4$ (the minimum wavenumber being $k_{\min }=1$ ), which means in physical space that the most energetic initial forcing waves have a length scale of one quarter of the box size.

Let us define the initial large-eddy turnover time as $T_{i n}=1 /\left(v k_{i}\right)$, where $(1 / 2) v^{2}$ is the initial kinetic energy. Let $v_{0}=v / \sqrt{3}$ be the rms velocity fluctuation in any direction of space. We will examine the formation and evolution of coherent vortices from $t=0$ up to about $10 T_{i n}$. 


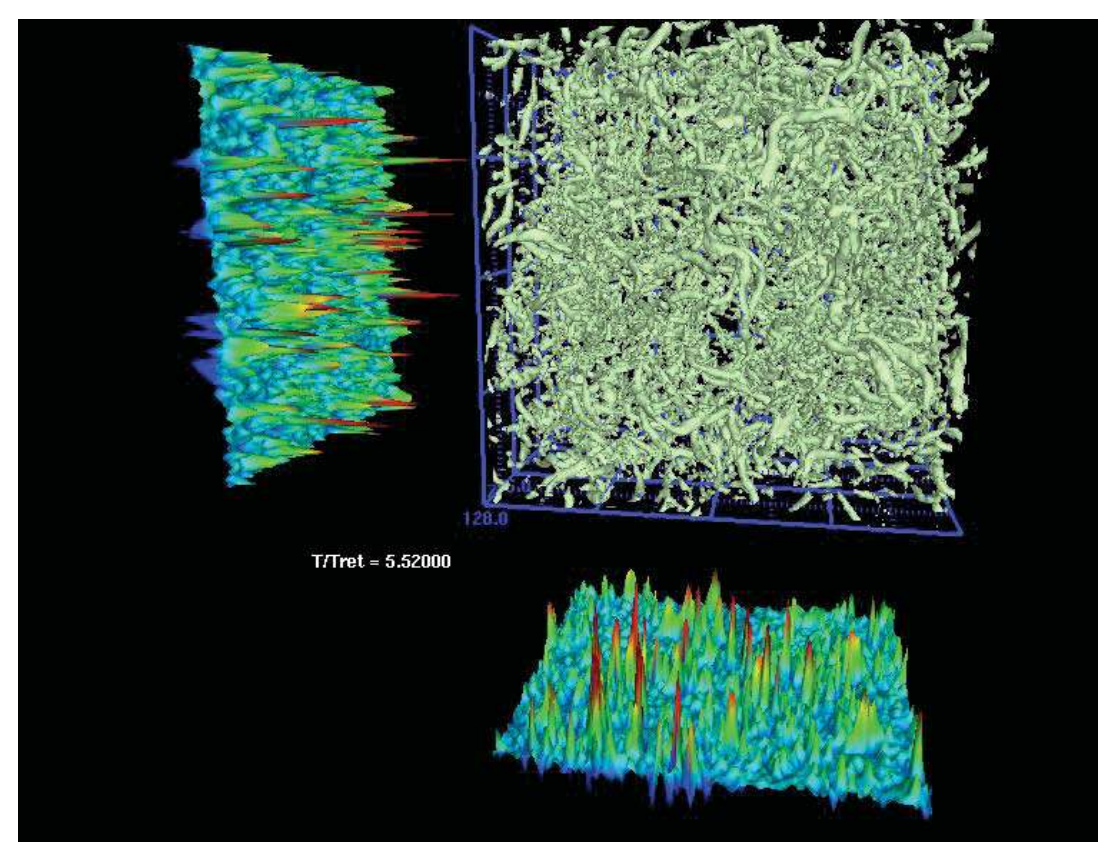

Figure 2. Animation of $Q$-isosurfaces at a given positive threshold; same conditions as in the animation of figure 1 .

The recognition of vortices will be based upon three criteria:

(i) isosurfaces of positive $Q$, where $Q$ is the second invariant of the filtered velocity-gradient tensor (see [9]), equal here to $\nabla^{2} P / 2 \rho$ for a uniform-density flow;

(ii) isosurfaces of the vorticity-vector norm and

(iii) isosurfaces of low macro-pressure $P$.

Our pressure is in fact of zero average, so that low pressure corresponds to negative values. The reader is referred to Dubief and Delcayre [9] for a review of certain vortex identification criteria in various flows without and with shear.

In the three animations to be presented now, the lower and left sides of the computational box are coloured by the value of the associated quantity on this side. In the pressure animation (see figure 1), the pressure threshold is fixed in time and chosen equal to $-2.1 v_{0}^{2}$ (pressure is divided by density). The threshold values for $P, Q$ and the vorticity are chosen empirically to give the best visual representation of vortices. The pressure animation starts with a few big low-pressure structures, in the form of sorts of billows and even bubbles, some of which seem to be attached to the billows. These structures are associated with the initial non-divergent Gaussian field. These big Gaussian structures evolve and interact in a complicated manner which is difficult to follow, in such a way as to become thinner and thinner. At $t=7$ they have nearly totally disappeared, at least as far as the particular threshold is concerned. Notice on the left-hand side of the box an initial low-pressure peak (due to initial conditions), whose intensity diminishes, then grows again at about $t=2$, then decreases. In the animation of figure 2 , where the threshold is $Q=300\left(v_{0} k_{\text {min }}\right)^{2}$, nothing is seen at the initial instant. Then the animation displays the progressive formation of tubes (much thinner than the pressure tubes) which have filled the space at $t=t_{c}=4$. After this time, one sees the rapid appearance of small-scale turbulence which seems to be due to the breakdown of larger-scale tubes in some regions of the flow, and is finished at $t=5$. Afterwards, one observes a superposition of large-scale and 


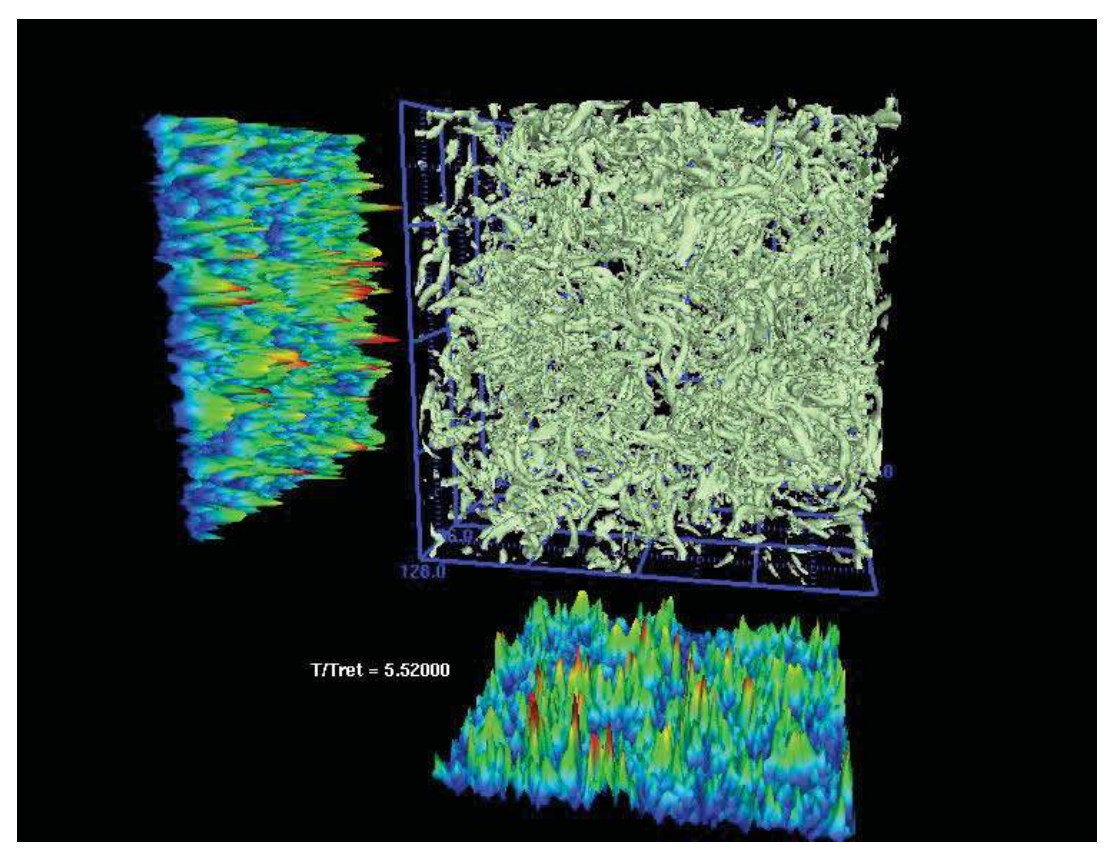

Figure 3. Animation of the vorticity modulus; same conditions as in the animation of figure 1.

fine-scale tubes, as well as other small scales not organized into tubes. Turbulence seems to be more intermittent in the sense that coherent structures occupy a smaller fraction of space. The animation of figure 3 shows in the same conditions the evolution of the vorticity modulus (at a threshold $30 v_{0} k_{\min }$ ) up to $t=10$. One sees hardly any difference when comparing with $Q$, and the formation of vortex sheets which by roll-up would generate the coherent vortices is not obvious. On the left-hand side of the box, and in contrast to the amplitude of pressure troughs, the intensity of high vorticity increases continuously during several turnover times.

One notices that the critical time $t_{c}$ discussed above is not far from the 'catastrophe' time $t_{*}$ arising in statistical theories of 3D isotropic turbulence, based on two-point closures of the eddy-damped quasi-normal Markovian (EDQNM) type: in the limit of zero molecular viscosity, the kinetic energy is conserved before $t_{*}$, and decays at a finite rate above. Still in this limit, the enstrophy blows up and becomes infinite at $t_{*}$, while a $k^{-5 / 3}$ kinetic-energy spectrum extending to infinity at high wavenumbers forms (see $[6,12,13]$ ). Figure 4 presents in our LES the time evolution of resolved kinetic energy and enstrophy. Here, energy starts dissipating slightly before 2 , while the enstrophy peaks at $t \approx 4.5$. This is due to the fact that we are in a LES, and was also noticed by Ackermann and Métais [14]. Such a behaviour is easily understandable in the framework of a typical spectral-eddy viscosity-based LES. Indeed, let us consider the resolved (for $k \leq k_{C}$ ) kinetic-energy spectrum evolution equation in the LES as

$$
\frac{\partial}{\partial t} E(k, t)=T_{<k_{C}}(k, t)-2 \nu_{t}\left(k \mid k_{C}\right) k^{2} E(k, t),
$$

where $\nu_{t}\left(k \mid k_{C}\right)$ is the spectral eddy viscosity, proportional to $\sqrt{E\left(k_{C}, t\right) / k_{C}}$, and $T_{<k_{C}}(k, t)$ the resolved kinetic-energy transfer, which is energy conservative in the sense that $\int_{0}^{k_{C}} T_{<k_{C}}(k, t) \mathrm{d} k=0$. We recall that no molecular viscosity is present in these simulations. The typical time evolution of the kinetic-energy spectrum may be split into three stages:

(i) a first 'inviscid' period where the spectrum spreads out towards high wavenumbers while 


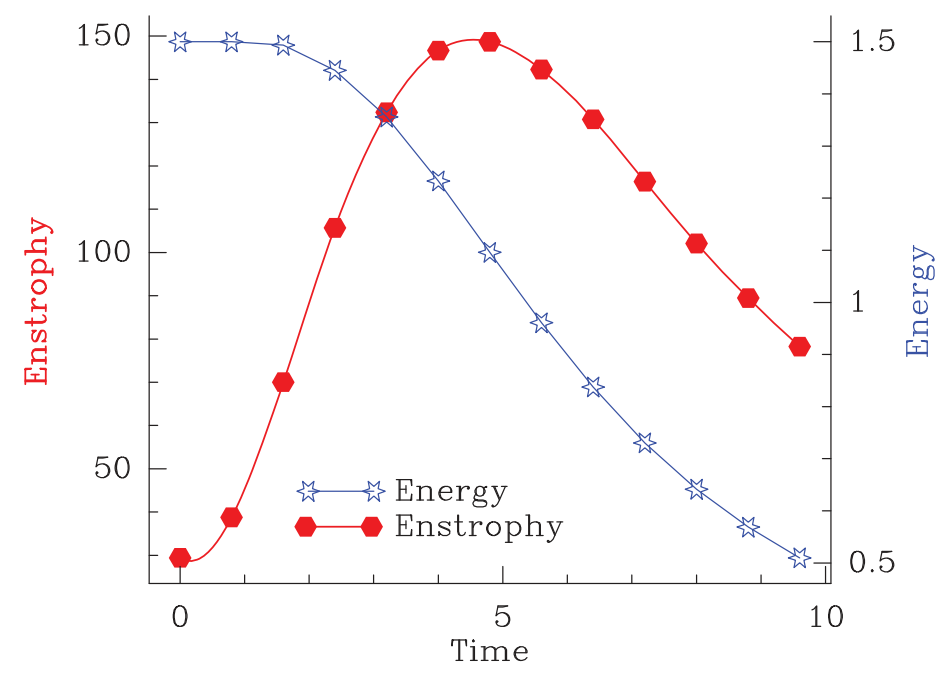

Figure 4. Time evolution of kinetic energy and enstrophy in the run $k_{i}=4,128^{3}$ modes. Time is given in initial large-eddy turnover times $T_{i n}$.

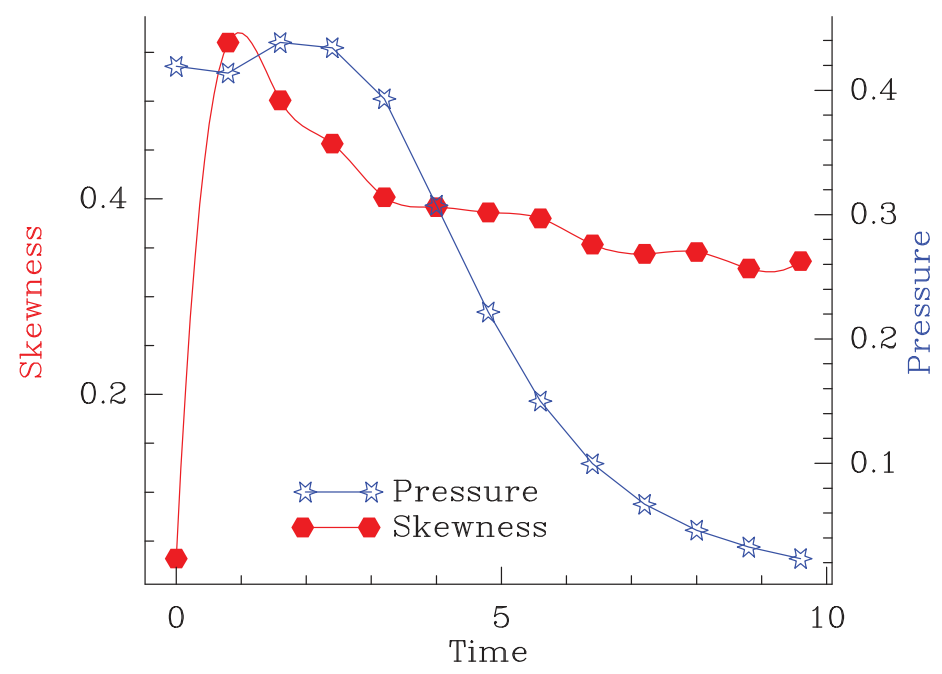

Figure 5. Time evolution of pressure variance and skewness in the run $k_{i}=4$, $128^{3}$ modes. Time is given in initial large-eddy turnover times $T_{i n}$.

$E\left(k_{C}\right)$ remains negligible in such a way that the eddy viscosity is not active, energy is conserved and enstrophy grows;

(ii) a second period where the energy spectrum at $k_{C}$ starts piling up to form a spectral range not too far from $k^{-5 / 3}$, which increases the enstrophy, while the eddy viscosity becomes active and energy decays;

(iii) a final stage where both the energy and enstrophy decay.

Figure 5 shows the time evolution of the pressure variance in the same run, together with minus the resolved skewness factor of the velocity derivative, a quantity which will be called here the skewness. The pressure variance starts decreasing slightly, then peaks at $t=2$, which seems to indicate the time of formation of large pressure tubes. The resolved skewness peaks at about 0.55 for $t \approx 0.9$, then decreases to 0.35 , a value comparable to 0.4 found experimentally 
in grid turbulence. More precisely, one has to clarify this notion of resolved skewness. Let us multiply equation (1) by $k^{2}$ and integrate from 0 to $k_{C}$. One obtains for the resolved enstrophy

$$
\frac{\mathrm{d}}{\mathrm{d} t} D(t)=\int_{0}^{k_{C}} k^{2} T_{<k_{C}}(k, t) \mathrm{d} k-2 \int_{0}^{k_{C}} \nu_{t}\left(k \mid k_{C}\right) k^{4} E(k, t) \mathrm{d} k .
$$

One can thus relate this expression to the resolved skewness $\bar{s}(t)$, defined here as the skewness factor of $-\partial \bar{u}_{1} / \partial x_{1}, u_{1}$ being any component of the velocity. Indeed, and in analogy with what is done in the general theory of isotropic turbulence, we have

$$
\left\langle\bar{\omega}_{i} \bar{\omega}_{j} \frac{\partial \bar{u}_{i}}{\partial x_{j}}\right\rangle=\left(\frac{98}{135}\right)^{1 / 2} \bar{s}(t) D^{3 / 2}
$$

The resolved enstrophy evolution equation can be easily written in physical space as

$$
\frac{\mathrm{d}}{\mathrm{d} t} D(t)=\left\langle\bar{\omega}_{i} \bar{\omega}_{j} \frac{\partial \bar{u}_{i}}{\partial x_{j}}\right\rangle+\operatorname{eddy}-\operatorname{visc} \ldots
$$

in which 'eddy - visc...' means the eddy-viscous contributions. Comparison with equations (2) and (3) permits us then to evaluate the eddy-viscous contributions as $-2 \int_{0}^{k_{C}} \nu_{t}\left(k \mid k_{C}\right) k^{4} E(k, t) \mathrm{d} k$, and to obtain

$$
\frac{\mathrm{d}}{\mathrm{d} t} D(t)=\left(\frac{98}{135}\right)^{1 / 2} \bar{s}(t) D^{3 / 2}-2 \int_{0}^{k_{C}} \nu_{t}\left(k \mid k_{C}\right) k^{4} E(k, t) \mathrm{d} k,
$$

with

$$
\bar{s}(t)=\left(\frac{135}{98}\right)^{1 / 2} D(t)^{-3 / 2} \int_{0}^{k_{C}} k^{2} T_{<k_{C}}(k, t) \mathrm{d} k .
$$

It is probable that for $t$ small enough, the resolved skewness converges towards the actual skewness of Navier-Stokes in the limit of zero molecular viscosity. The sharp initial growth of the skewness is encouraging in terms of finite-time singularities. Indeed, Lesieur ([6, pp 190191], has shown a theorem stating in the Euler limit that, if the skewness grows from zero, then decays up to strictly positive values, the enstrophy blows up at a finite time. We mention that the EDQNM study of Lesieur and Ossia [13] shows that, in the limit of zero viscosity, the skewness grows to a maximum value of 1.132 , reached at $t=4.1$, then abruptly drops to a plateau of 0.5 corresponding to a self-similar behaviour of the energy spectrum in inertial and dissipative scales.

We present now LES with $k_{i}=2$ and $128^{3}$ Fourier modes. We display in figure 6 (left) the kinetic-energy and normalized-enstrophy $D(t) / D(0)$ time evolution in units of $T_{i n}$, with a comparison to the $k_{i}=4$ case. The kinetic energy is conserved up to slightly later than 2 , and the enstrophy peaks now at $t=5.5 T_{\text {in }}$ instead of 4.5 . The maximal relative enstrophy is $45 \%$ higher, which shows that vorticity stretching is more efficient in this case, due to the longer available ultraviolet range in Fourier space. Figure 6 (right) presents the same data in units of time $D(0)^{-1 / 2}$, which are an alternative way of measuring time ${ }^{3}$. Figure 7 is the equivalent of figure 6 for the skewness and pressure variance. The skewness peaks now at $\approx 0.8$ reached at a time slightly higher than $T_{i n}$, then drops, slightly oscillates and saturates at $\approx 0.4$. The pressure variance has a marked peak at $3 T_{i n}$. It is not clear yet whether the skewness drop at $t \approx T_{i n}$ in both cases $k_{i}=2$ and 4 is due to the spectral-cut-off effects of the LES, or is inherent to Navier-Stokes equations in the limit of zero viscosity.

3 We note for instance that in the constant-skewness model based on equation (5) where the eddy-viscous term is neglected, the enstrophy blows up at a time of $D(0)^{-1 / 2} /\left(0.425 s_{0}\right)$, where $s_{0}$ is the value of the constant skewness (see [6, p 189]). This yields $5.9 D(0)^{-1 / 2}$ for $s_{0}=0.4$, and $6.7 D(0)^{-1 / 2}$ for $s_{0}=0.35$. 

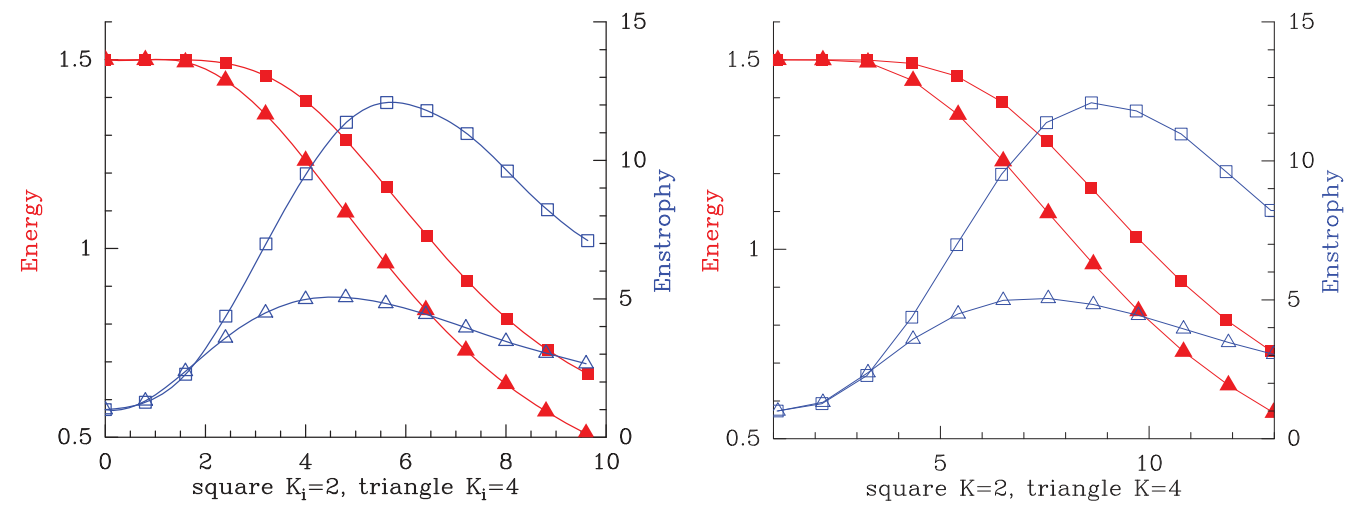

Figure 6. Compared time evolution of kinetic energy and relative enstrophy for runs $\left(k_{i}=2,128^{3}\right)$ and $\left(k_{i}=4,128^{3}\right)$. Units of time are $T_{i n}$ (left) and $D(0)^{-1 / 2}$ (right).
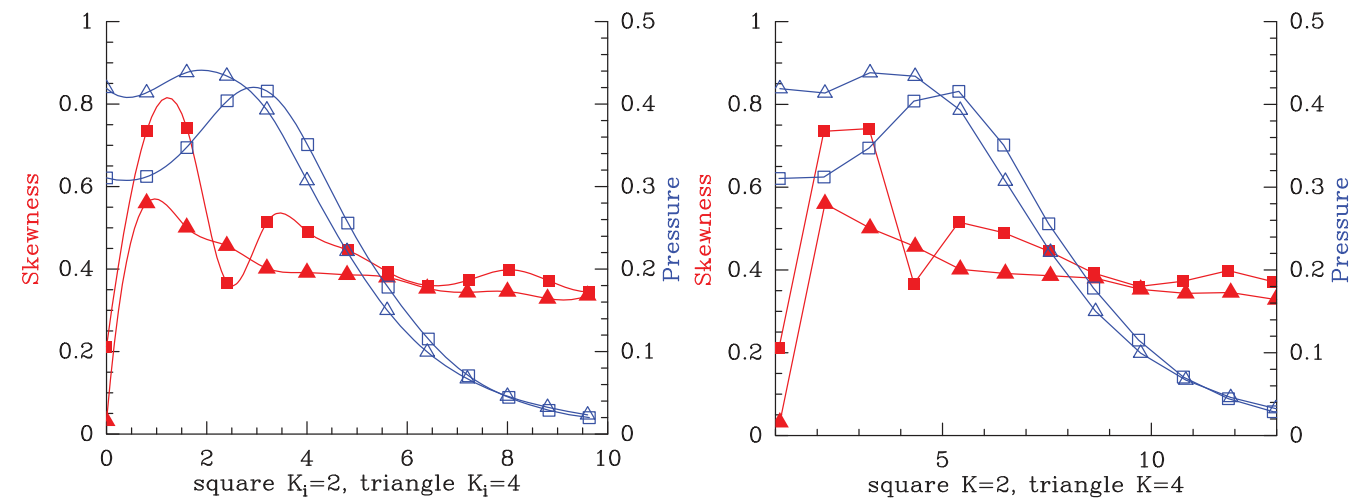

Figure 7. Compared time evolution of skewness and pressure variance for runs $\left(k_{i}=2,128^{3}\right)$ and $\left(k_{i}=4,128^{3}\right)$. Units of time are $T_{i n}$ (left) and $D(0)^{-1 / 2}$ (right).

\section{Boundary layer developing on a flat plate}

We present here LES using the filtered structure function model of a quasi-incompressible $\left(M_{\infty}=0.3\right)$ boundary layer (without pressure gradient) of an ideal gas developing spatially over an adiabiatic flat plate with a low level of upstream forcing, as a continuation of the work of [15]. The numerical methods are MacCormack-type finite differences (second order accurate in time, and fourth order in space for the nonlinear terms). Periodicity is assumed in the spanwise direction, and non-reflective boundary conditions are prescribed in the vertical open boundaries. Free-slip boundary conditions are assumed on the upper horizontal plane of the computational domain, which is rejected to infinity thanks to a proper mapping. The upstream Reynolds number based on the displacement thickness is $R_{\delta_{1}}=1000$. The resolution at the wall is $y^{+}=1$. The upstream conditions are generated from nonlinear parabolized stability expansion (PSE) methods [16] thanks to a code provided by Airiau et al [17]. On this upstream state, one superposes a 3D white noise of amplitude 0.2 times the amplitude of the PSE perturbation. One can thus reach harmonic (K-type) or subharmonic (H-type) upstream fields. The animation of figure 8 presents the transition for these two cases. There are in fact big $\Lambda$ vortices lying on the wall and travelling with the flow, inducing low- and high-speed streaks extremely well 
(a)

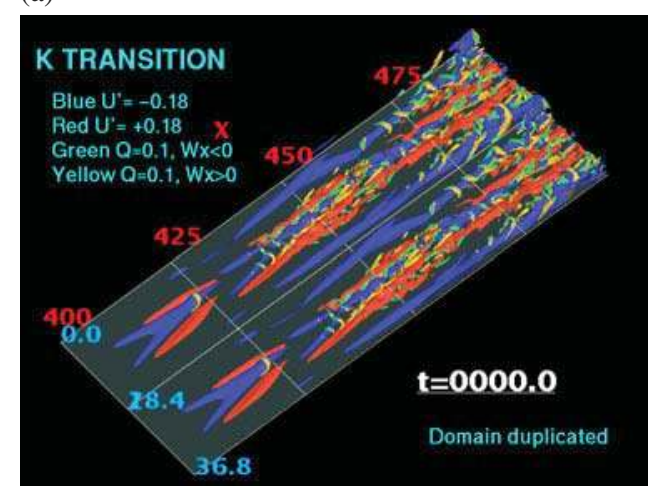

(c)

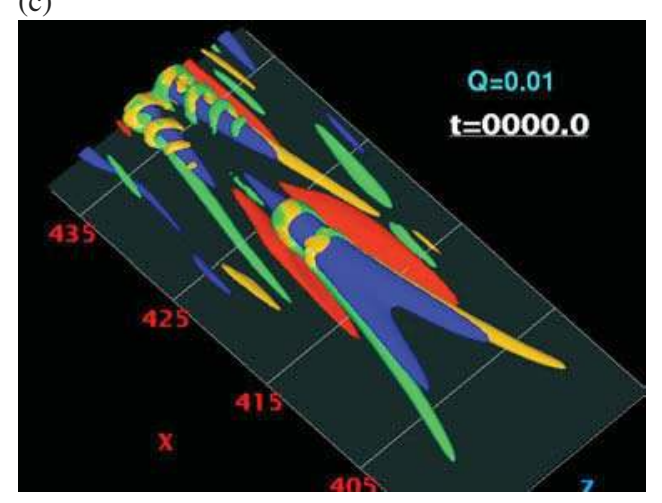

(b)

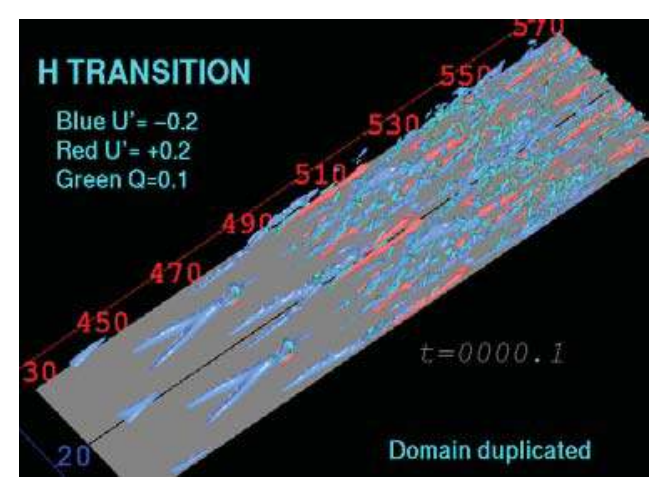

(d)

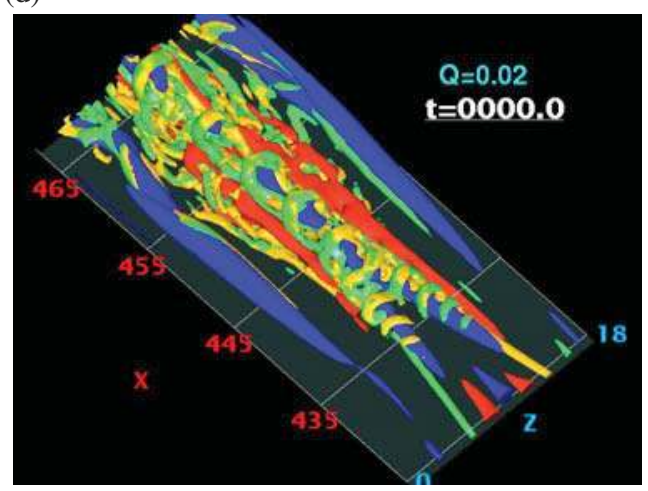

Figure 8. $u^{\prime}$ and $Q$ in the animation of the transition in the spatial boundary layer over a flat plate at Mach 0.3 ((a) harmonic forcing animation, (b) subharmonic forcing animation, (c) zoom with $Q=0.01$ animation, (d) zoom with $Q=0.02$ animation).

correlated with the vortices (see below). These streaks are not longitudinal since they follow the $\Lambda$ 's legs. Downstream of $\approx 440 \delta_{i}$, the streaks become purely longitudinal. This is accompanied by the fast shedding of small hairpin vortices ejected from the tip of the $\Lambda$ s, as indicated by $Q$-isosurfaces. A change of threshold shows for these hairpins a very complex structure, since they seem to originate from both sides of the big $\Lambda$ vortex. Similar patterns have been observed experimentally by Christensen and Adrian [18].

We have checked that the rms longitudinal velocity component at a downstream distance such that $R_{\delta_{1}}=1670$ is in between the DNS results of Spalart [19] at Reynolds numbers of 1000 and 2000. The animation of figure 9 shows both transition and developed turbulence in the same simulation (harmonic forcing). During transition, we see isosurfaces of $u^{\prime}$ (figure 9(a)), with high-and low-speed streaks very well correlated with the big $\Lambda$ 's legs, isosurfaces of longitudinal vorticity (figure 9(b), with 'anti-vorticity', that is vorticity of opposite sign, close to the wall, due to the no-slip condition at the boundary) and isosurfaces of fluctuating spanwise vorticity (figure $9(\mathrm{c})$ ). The latter is proportional at the wall to the drag-coefficient fluctuation. The animations of figures $9(\mathrm{~d})$ and (e) show respectively high- and low-speed streaks and low pressure in the developed turbulence further downstream. The low-speed streaks are lifted by what seems to correspond to the passage of quasi-longitudinal vortices which are well known to exist in boundary layers and in channels (see [9] in the channel case). The pressure displays only the tips and some branches of these vortices. 
(a)

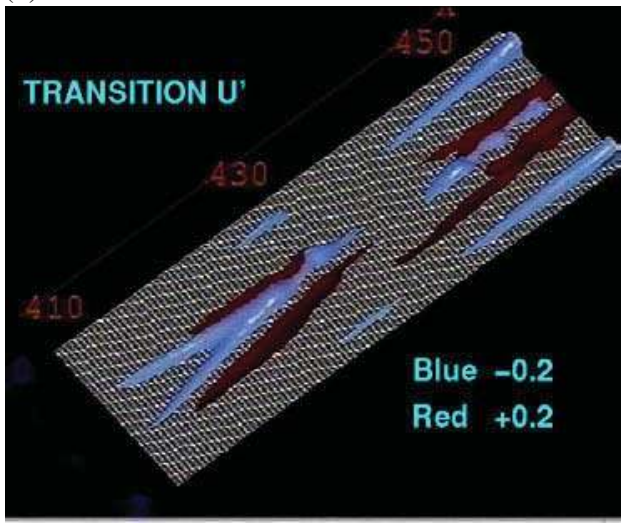

(c)

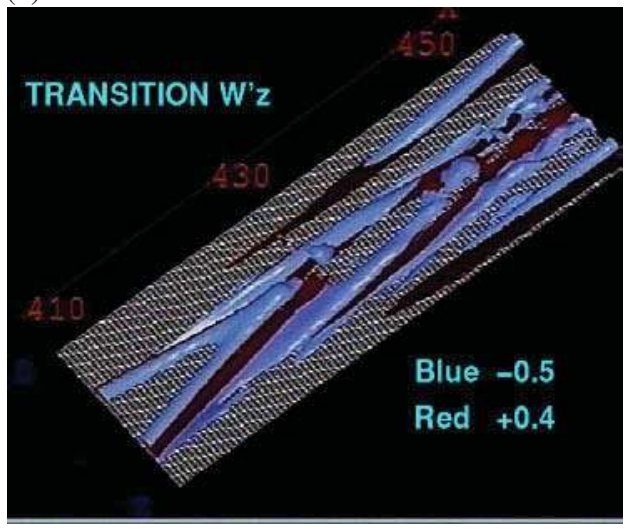

(b)

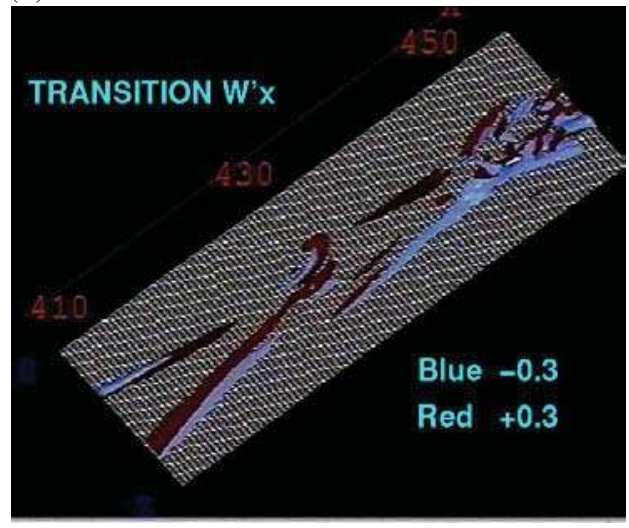

(d)

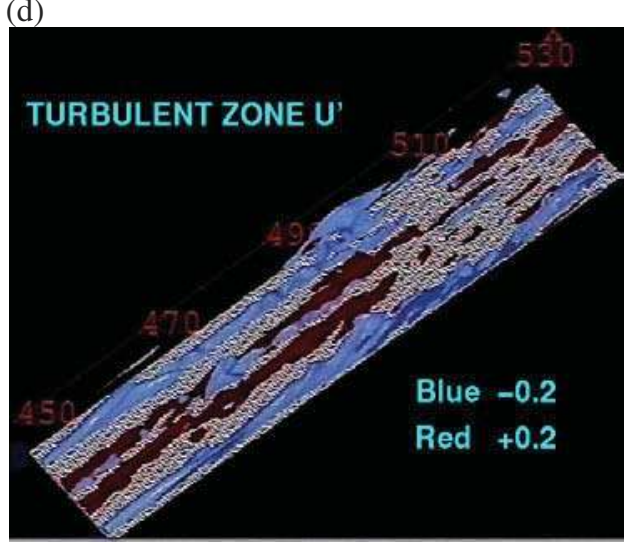

(e)

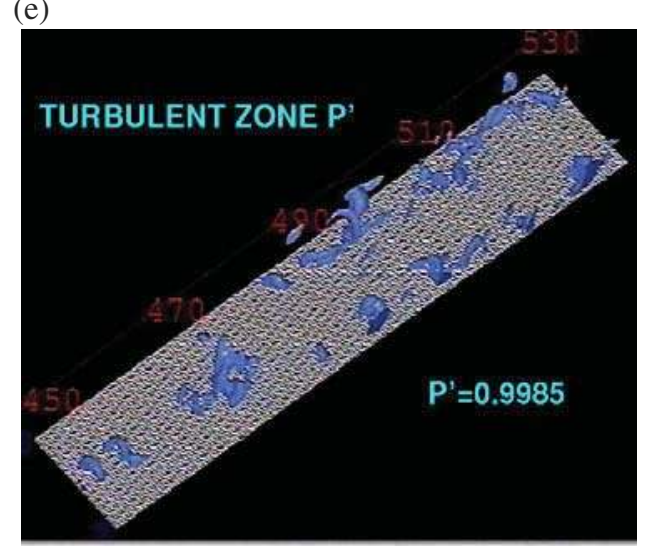

Figure 9. Animation of the spatial boundary layer over a flat plate at Mach 0.3 (harmonic forcing); transition ((a) $u^{\prime}$ animation, (b) $\omega_{x}^{\prime}$ animation, (c) $\omega_{z}^{\prime}$ animation); developed turbulence region ((d) $u^{\prime}$ animation, (e) $P^{\prime}$ animation.)

\section{Backward-facing step}

The same compressible LES code as for the above boundary layer is now used to simulate the flow above a straight backward-facing step (see [20] for more details). The Reynolds number based on the step height $H$ is $U_{0} H / \nu=5100$ (where $U_{0}$ is the incoming velocity at the top of the domain) as in the incompressible DNS of [21]. The domain dimension is $18 H \times 6 H \times 4 H$. The 
(a)

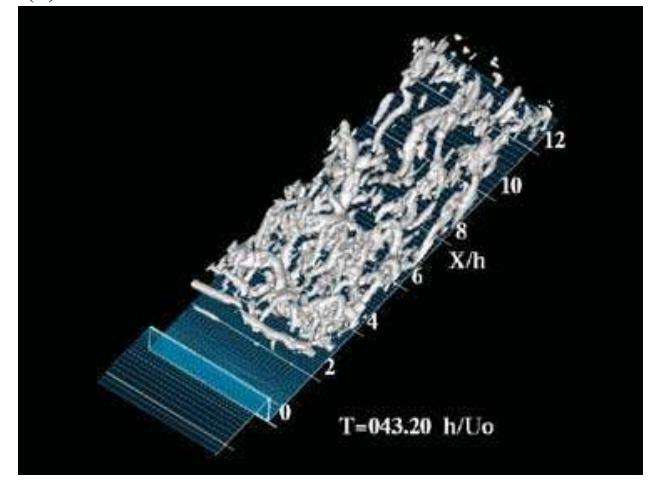

(b)

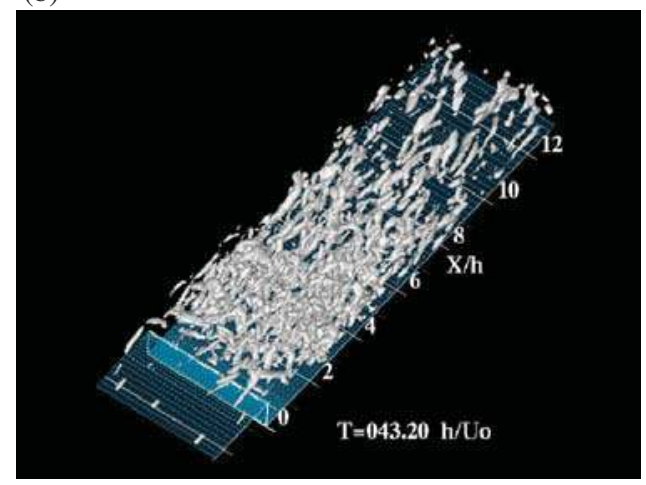

Figure 10. Perspective view of the back-step: left animation, noisy upstream velocity; right animation, realistic upstream velocity.
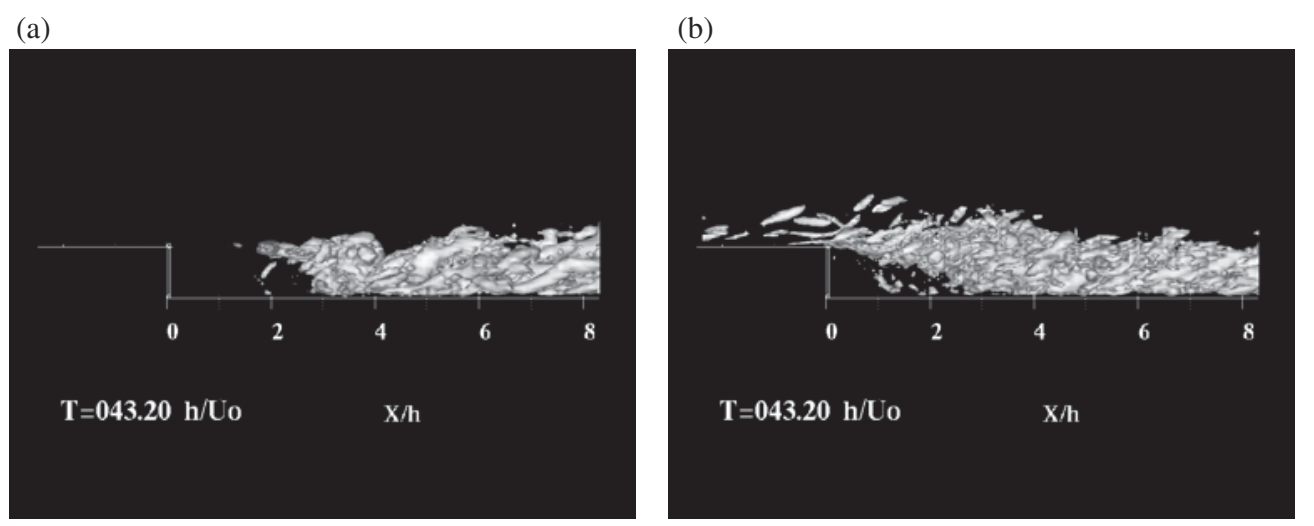

Figure 11. Side view of the back-step: left animation, noisy upstream velocity; right animation, realistic upstream velocity.

momentum thickness of the boundary layer upstream of the step is $0.13 H$. The first grid point in the direction normal to the wall is at a distance of 1.3 wall units relative to the upstream turbulent boundary layer. The Mach number is 0.3. Periodicity is assumed in the spanwise direction, and the boundary condition at the top of the domain is non-reflective. This makes an important difference with respect to the uniform-density DNS of [21] when free-slip conditions are taken there. The total number of grid points is 670000 . The animations of figures 10 and 11 show $Q$ isosurfaces (threshold $0.6 U_{0}^{2} / H^{2}$ ) for two classes of upstream conditions modelling the boundary layer upstream of the step:

(a) a mean velocity profile corresponding to Spalart's boundary-layer DNS [19] perturbed by a $3 \mathrm{D}$ white noise of intensity $1.5 U_{0}$ and

(b) a precursor calculation, with a more realistic time-dependent velocity field generated through an extension to the compressible case of the method developed in [22]. In this case, one can see on the right of figures 10 and 11 quasi-longitudinal vortices propagating before the step.

In figure 10 left, one sees the regular shedding of straight quasi-2D Kelvin-Helmholtz vortices, which appear at a distance of 1.5-2H downstream of the step. They undergo helical pairing, and transform into big $\Lambda$ vortices which impinge the lower wall and are carried away from the step. 


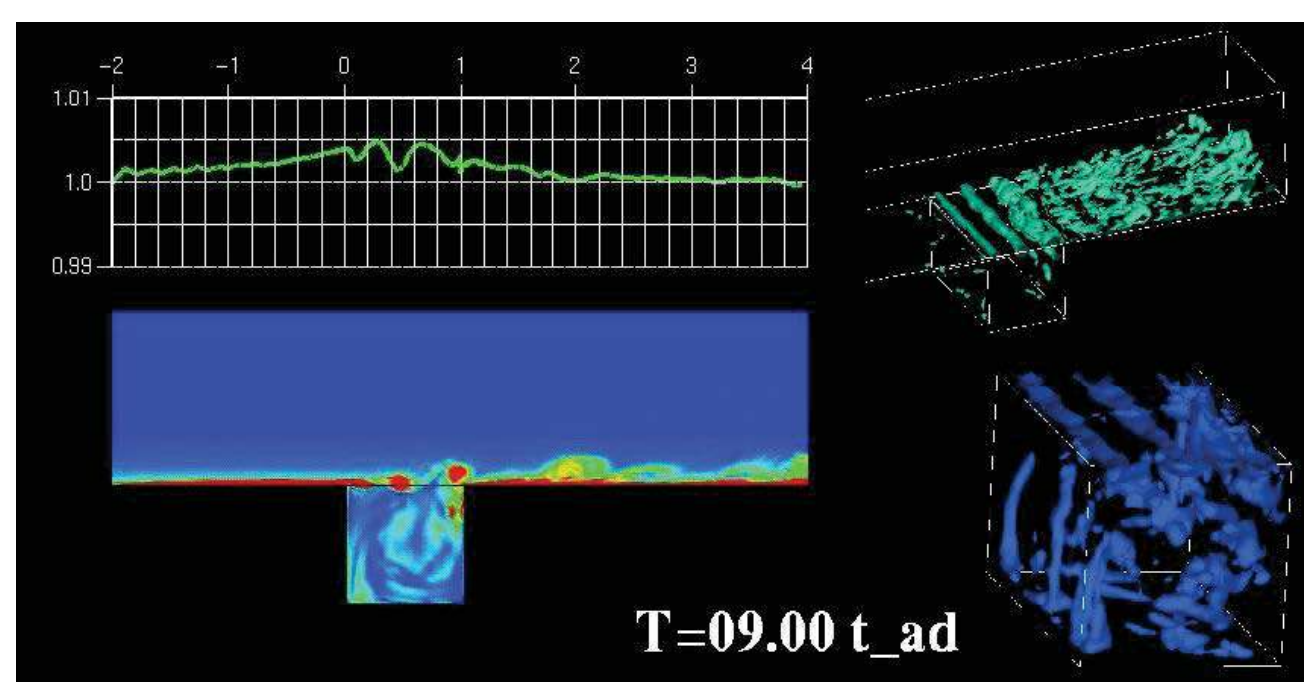

Figure 12. Animation of the flow above a $2 \mathrm{D}$ square cavity; vertical section of vorticity norm and pressure at the wall; 3D map of $Q$; zoom of $Q$ in the cavity.

Figure 10 right shows qualitatively the same events, but vortices appear very close to the step, and the flow is much more 3D. Helical pairing seems to be triggered by the passage of upstream longitudinal vortices passing above the step. The side views of figure 11 confirm that the flow reattaches sooner in this case than in the noisy case. This is confirmed by the determination of the reattachment length, which is $5.80 \mathrm{H}$ for (a) and $5.29 \mathrm{H}$ for (b). The latter value is quite different from the value of $\approx 6.1 \mathrm{H}$ found by [21] with equivalent upstream conditions. We think that this discrepancy may be attributed to the differences in the boundary conditions above and downstream of the computational domain. Indeed, non-reflective boundary conditions pose problems at low Mach numbers, since incoming characteristics should not be cancelled. This creates pressure blockage which is certainly responsible for the error in the reattachment length. However, we think that the LES gives qualitatively here the right vortex dynamics, which is dominated by inviscid instabilities such as Kelvin-Helmholtz and helical pairing. Let us mention that analogous big Lambda-shaped vortices can be found in a lot of separated flows.

\section{Two-dimensional square cavity}

Here, one studies a weakly compressible flow (Mach 0.1) above a flat plate, passing over a square cavity. The latter is infinite in the spanwise direction. The Reynolds number based on the velocity at infinity $U_{0}$ and the cavity depth $H$ is 270000 . The geometric configuration is seen at the beginning and the middle of the animation presented in figure 12. The spanwise extent of the cavity is $2 H$, and periodicity is assumed in the spanwise direction. The total number of grid points is 688000 . The grid is refined close to the wall and in the region of strong shear between the edges. The upstream conditions correspond to a mean velocity of a turbulent boundary layer upon a flat plate given by a power law of the form $u / U_{0}=(y / \delta)^{1 / 7}$ (see [23]). The total stress $\tau_{0}$ is defined by Blasius' empirical relation

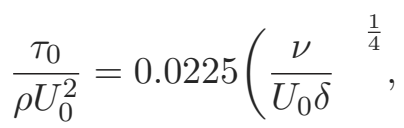

which allows us to define the friction velocity. To this profile is superposed close to the wall a white noise of intensity $5 \%$. We have $\delta=0.4 H$. The numerical methods are the same as in 


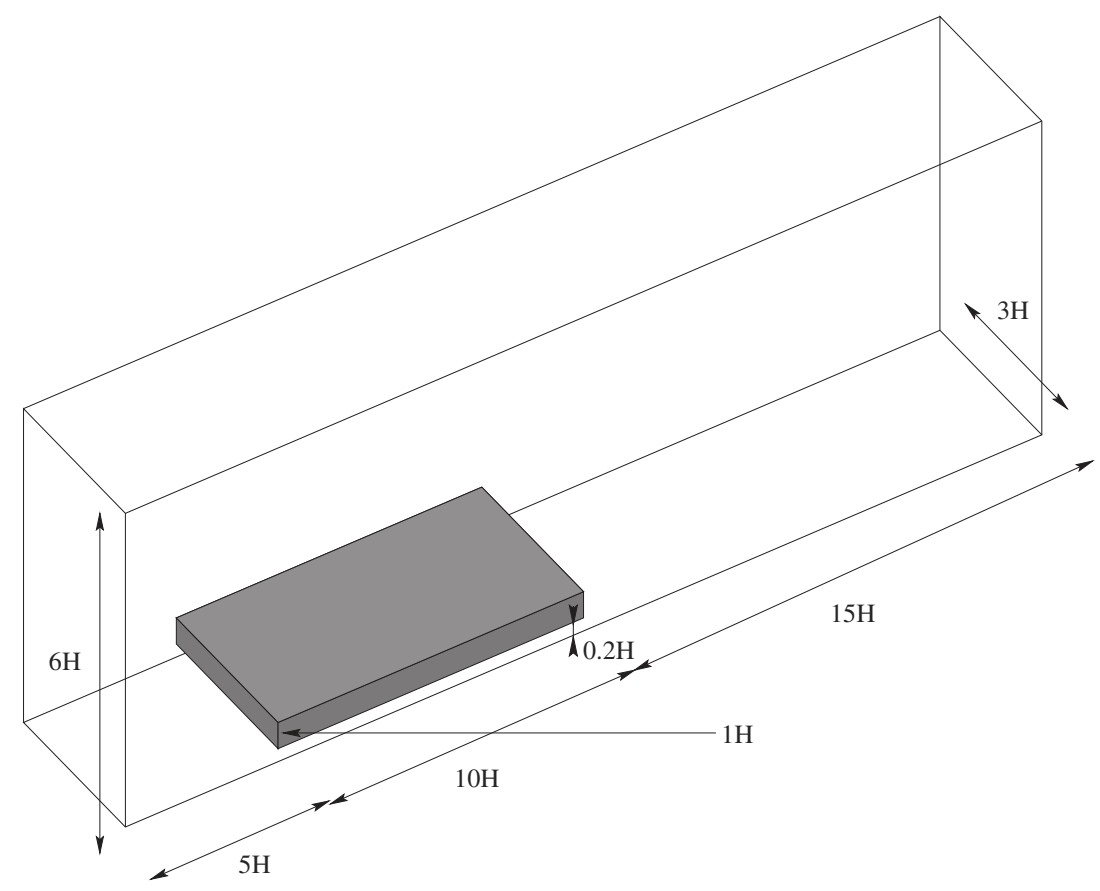

Figure 13. The $2 \mathrm{D}$ rectangular obstacle.

the compressible situations studied above. The animation of figure 12 presents first a vertical section of the vorticity norm, together with the pressure signal on a line $y=0$ (that is, on the upstream and downstream walls and the line joining the two edges of the cavity). The vorticity norm reveals the passage of Kelvin-Helmholtz type vortices shed behind the first back-step and impinging the second edge of the cavity. The pressure signal at the wall has a high frequency associated with these vortices, and a much lower one which is certainly due to the recirculation of the flow within the cavity. One can observe the four kinds of interaction proposed by [24],

- vortices escaping completely from the cavity (complete escape),

- vortices escaping partially from the cavity (partial escape),

- vortices completely captured by the cavity (complete clipping) and

- vortices partially captured by the cavity (partial clipping),

which is an argument in favour of the reality of our 'LES vortices'.

The second part of the animation deals with a perspective view of $Q$, confirming the generation of quasi-2D vortices behind the first edge. Since the length to travel is short, they do not have time to three-dimensionalize much. When impinging the second edge, they transform into very coherent big $\Lambda$ vortices which become arches by raising their tips, due to self-induction. The third part of the animation is a zoom of $Q$ inside the cavity. One sees on the top KelvinHelmholtz vortices passing by. In fact, the recirculation in the cavity produces a lot of longitudinal vortices and turbulence, maybe through Görtler instability due to the curvature of the flow in the cavity. These longitudinal structures have also been observed experimentally in [25].

\section{6. $2 \mathrm{D}$ rectangular obstacle with wall effect}

We present now a LES of a weakly compressible flow of Mach 0.2 around a 2D (infinite in the spanwise direction) rectangular obstacle of thickness $H$, length $10 H$, parallel to a wall and 


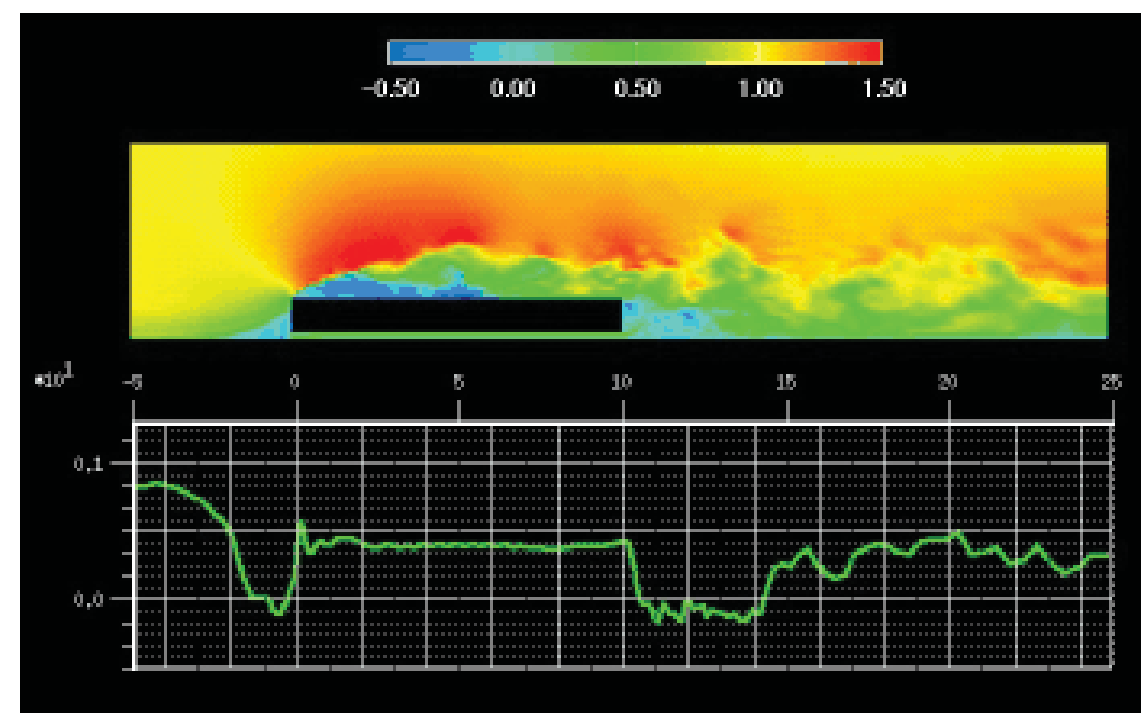

Figure 14. Animation of longitudinal velocity in a vertical plane parallel to the mean flow.

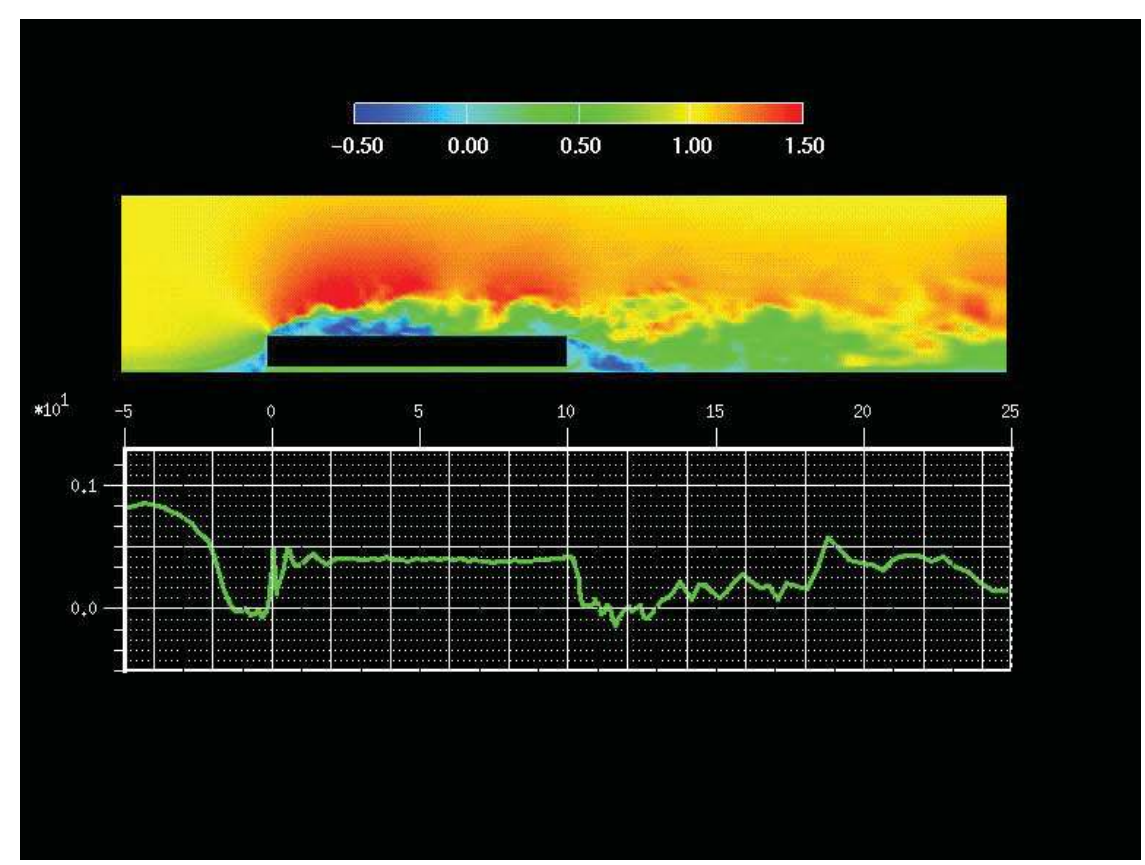

Figure 15. Instantaneous vertical section of longitudinal velocity, with this velocity at the altitude $y=0.1 H$.

located at a height of $0.2 \mathrm{H}$ above it. In fact periodicity is assumed in the spanwise direction. The Reynolds number based on the velocity at infinity $U_{0}$ and $H$ is 165000 . The geometric configuration is presented in figure 13. The spanwise width of the domain is $3 H$. The numerical compressible methods are still the same. The upstream velocity is identical to that for the above 2D square cavity, with $\delta=0.1 H$.

The grid of 1542000 points is split into four subdomains. The first point above the upper wall is at $y^{+}=15$, which is not too bad but is of course insufficient to properly resolve the 


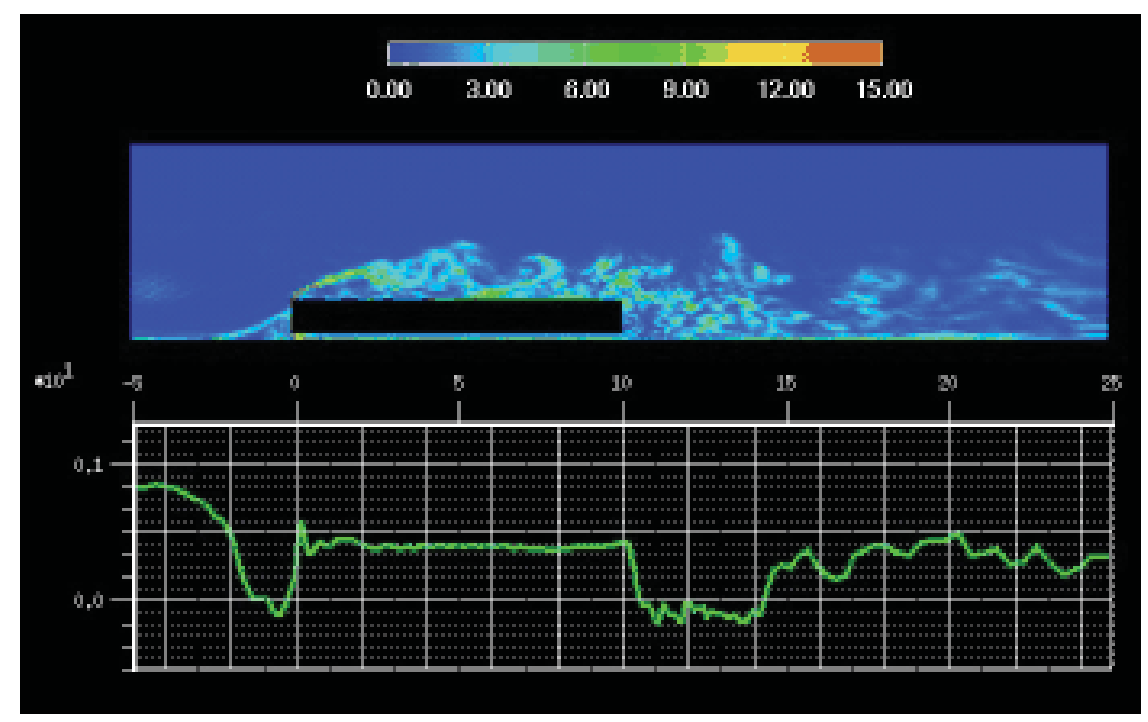

Figure 16. Animation of vorticity norm in a vertical plane parallel to the mean flow.

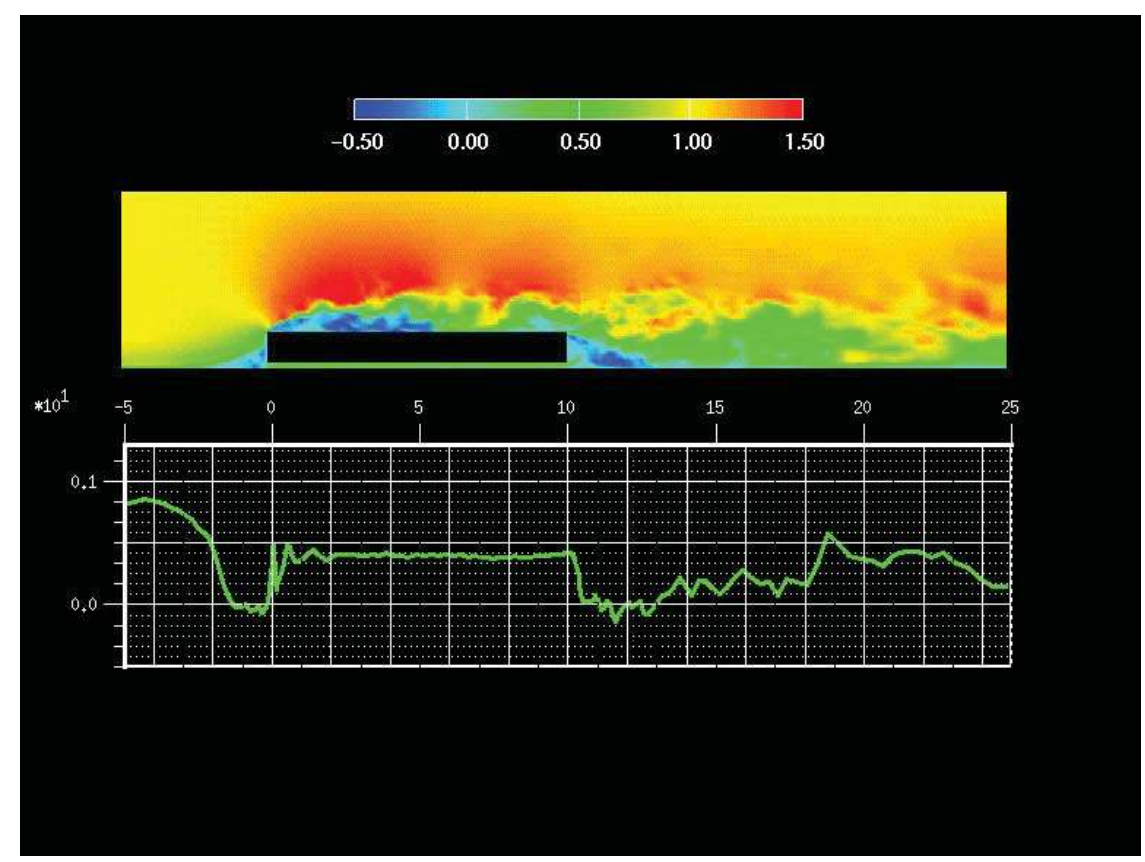

Figure 17. Instantaneous vertical section of the vorticity modulus, still with $u(y=0.1)$.

turbulent upstream boundary layer. However, the flow upstream will be dominated by a 'risingmarch effect', and might not be very appropriate to a proper description of the upstream turbulent boundary layer. On the other hand, boundary layers developing above the upper and downstream walls are much thicker than a classical boundary layer on a flat wall without pressure gradient, which might justify the chosen resolution.

The animations show the main characteristics of the flow very well. The animation of figure 14 is a cut, in the vertical middle plane parallel to the mean velocity, of the instantaneous 


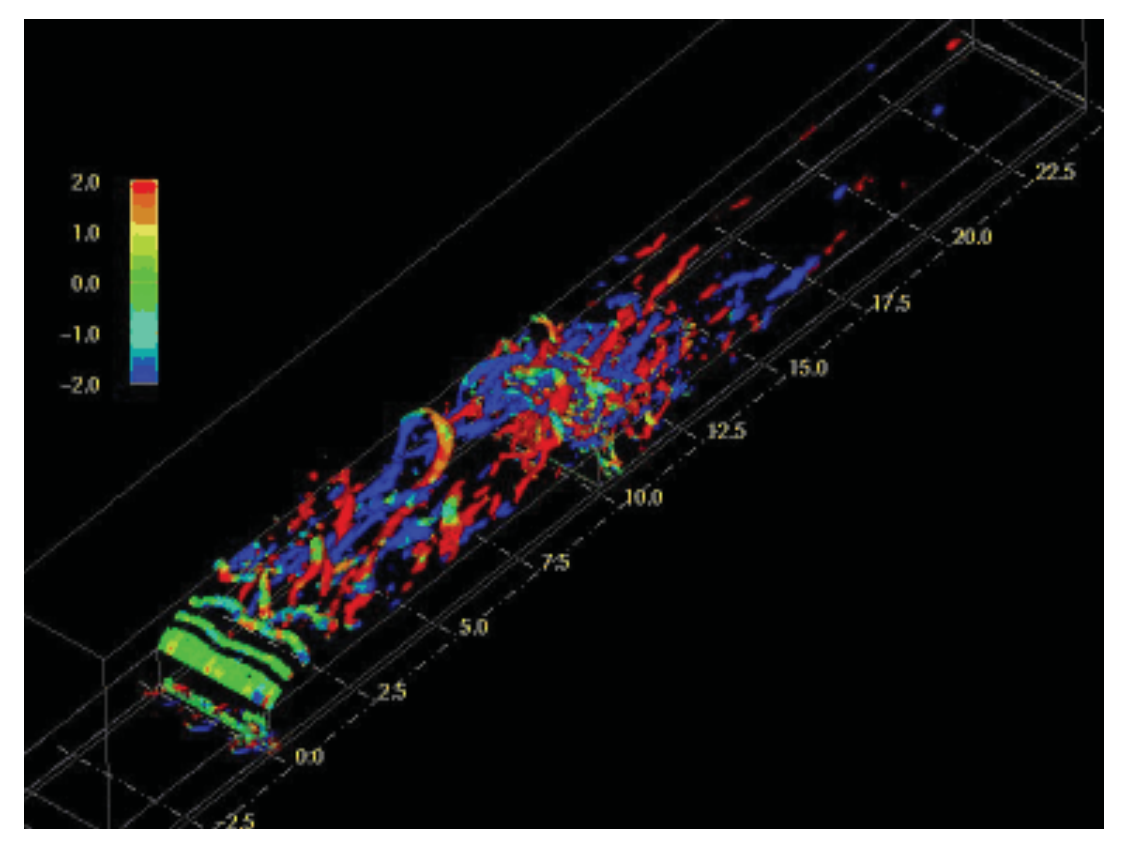

Figure 18. Animation of $Q$ and longitudinal vorticity.

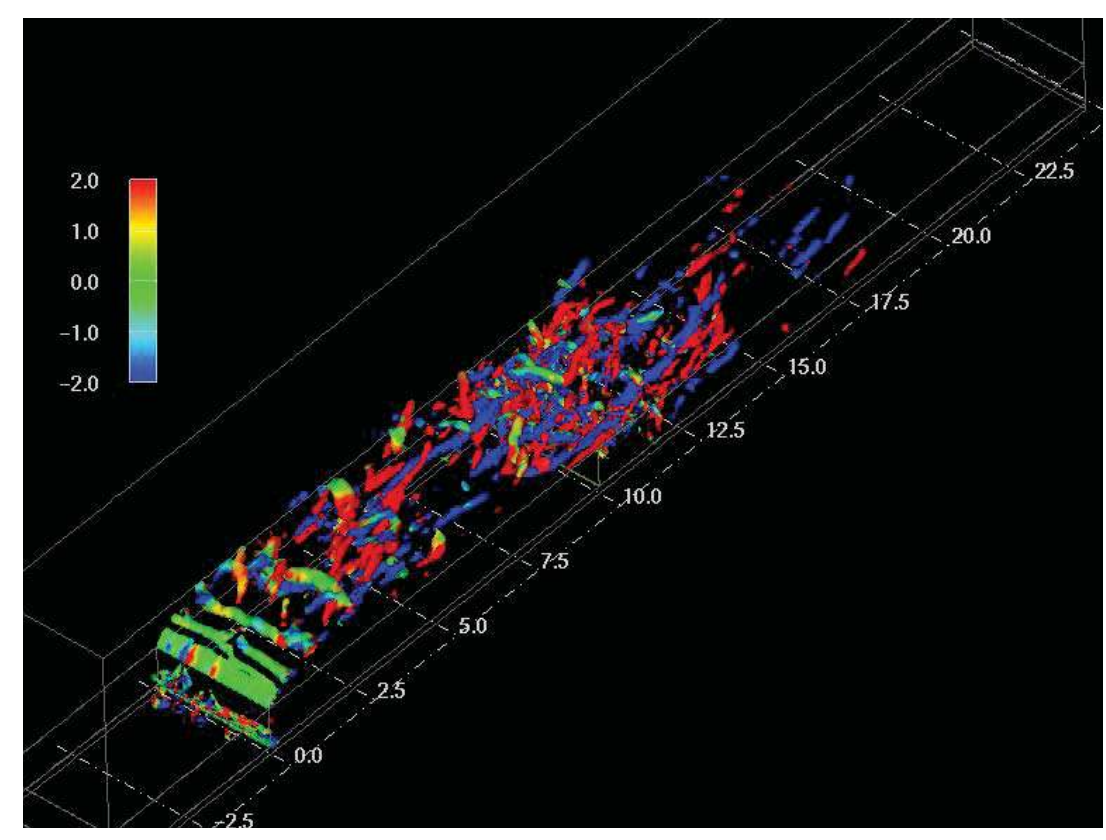

Figure 19. $Q$ and longitudinal vorticity.

longitudinal velocity, together with the same velocity on a line $y=0.1 H$. This is in the middle of the slot between the body and the ground. An instantaneous image is presented in figure 15, at a time whose choice will be made explicit later. The animation of figure 16 is a cut of the vorticity norm in the same plane, still with the longitudinal velocity close to the ground. Figure 17 is an image of this animation, corresponding to the same time as figure 15 . The animation of figure 18 is a global view of the flow, and represents iso- $Q$ surfaces coloured by longitudinal vorticity (positive, red; negative, blue). Figure 19 is an instantaneous view of this 
animation at the same time as above. These animations show clearly the main stages of the upstream-downstream flow evolution.

(A) The fluid impacts the rising step.

(B) A big recirculation bubble is created on the upper side of the plate, as in the experiments of $[26]$.

(C) Kelvin-Helmholtz type vortices are shed from the leading edge made by the upstream ridge and undergo dislocations, transforming into big $\Lambda$ vortices.

(D) These vortices reattach on the upper wall thanks to the downflow generated by the recirculation bubble.

(E) They detach on the trailing edge made by the downstream ridge and impinge the ground.

(F) Afterwards they keep on their way downstream, as in the reattached arch eddies already seen above for the back-step and $2 \mathrm{D}$ cavity cases.

The animation of figure 20 is a front view of $Q$ and longitudinal vorticity. It corresponds to figure 21. One sees very clearly strong longitudinal hairpins shed from time to time above the upper edge. In fact, the particular time of the preceding figures was chosen in order to outline this phenomenon. These hairpins are the source of perturbations which destabilize further downstream Kelvin-Helmholtz vortices shed behind the upstream edge. The animation of figure 22, corresponding to figure 23, is a zoom of positive $Q$ isosurfaces in the back-step region (stage (D) above). The flow resembles the back-step and cavity in the reattachment region. One sees vortices passing by as a flight of big migratory birds. They also look like quasi-longitudinal vortices observed in boundary layers on a flat plate without pressure gradient, but are much bigger. We note also in the animation of figure 22 the fact that the flow downstream of the slot collides close to the wall with the upstream flow coming from the recirculation bubble behind the back-step, and rises. We show now in figures 24-28 temporal and spanwise averages of the following quantities: longitudinal velocity, rms longitudinal velocity, vertical velocity, pressure and rms pressure. They indicate the existence of a large recirculating zone behind the leading edge, where kinetic energy is maximum and pressure minimum. On the other hand, pressure is maximum on this edge. It is also very high at the entrance of the slot under the obstacle. We have determined the position of recirculation bubbles, drawing the lines of zero-mean longitudinal velocity in figure 29. One clearly sees the small recirculation zone upstream of the rising step, visible also in figure 24 and the animation corresponding to figure 15. One also sees the big upper recirculation zone of length $7 \mathrm{H}$. It is obvious that the recirculation zone behind the back-step is much reduced with respect to a simple back-step, since the reattachment point is approximately $3.4 \mathrm{H}$ downstream of the step (instead of $6-7 H$ for a regular step). Notice also between the back-step and this recirculation zone the existence of a second recirculation pocket due to the existence of a flow in the slot. Remark finally in the figure 14 animation a sort of exchange between these downstream instantaneous recirculation pockets (seen as blue regions). Figure 30 displays the average eddy viscosity, which is very important in the recirculation pocket above the obstacle, and quite significative behind the back-step. This confirms a proper behaviour of our subgrid model, since the eddy viscosity should dissipate efficiently in regions of strong kinetic energy, otherwise there would be an excessive local accumulation of energy which might yield blow-up of the numerical simulation.

We have put in the computational space $63 \times 30$ numerical probes recording the various signals as a function of time. They correspond to 63 positions in the plane $x, y$, which are sketched in figure 31 and are recorded in the $30 z$-planes of the domain. At these points, we have determined the time-frequency spectra of pressure and velocity, averaged in the spanwise direction. Figure 32 represents a perspective view of 22 of these pressure spectra (normalized by the highest value), in which the vertical axis has been stretched. We recall that these 


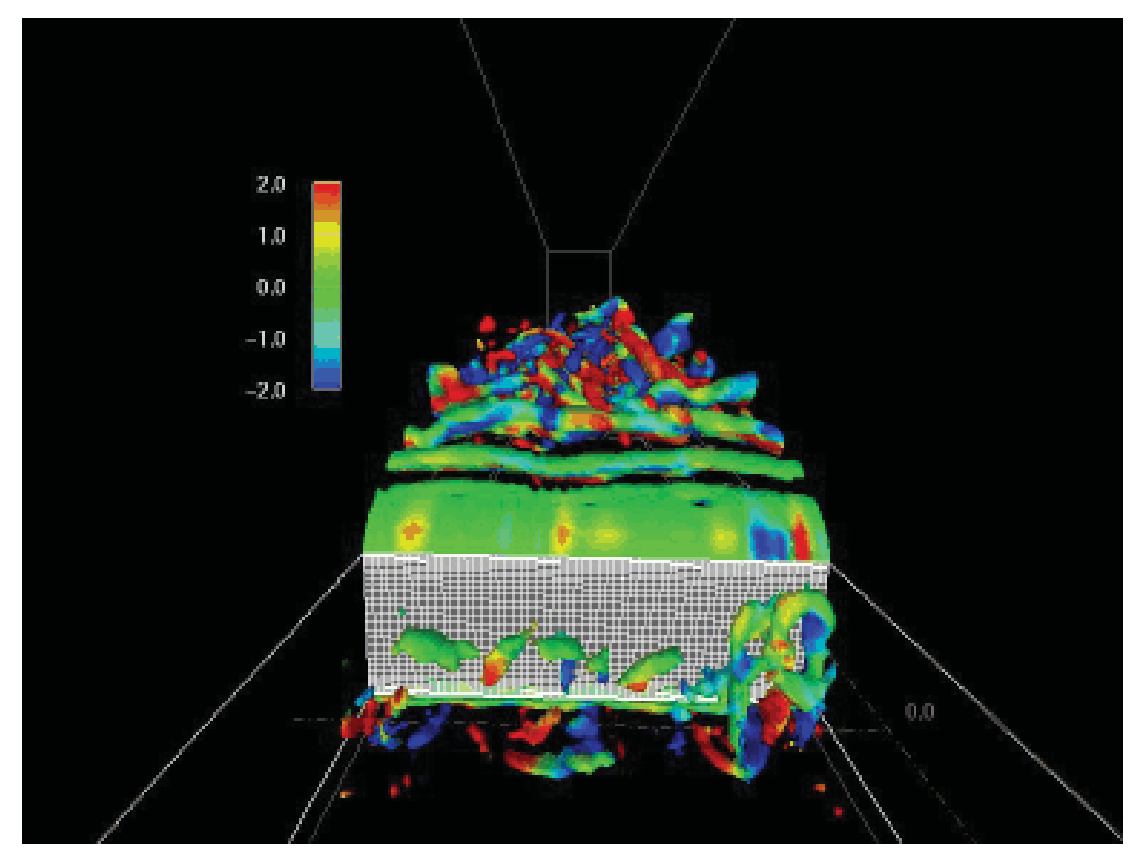

Figure 20. Animation of the front view of $Q$ and longitudinal vorticity.

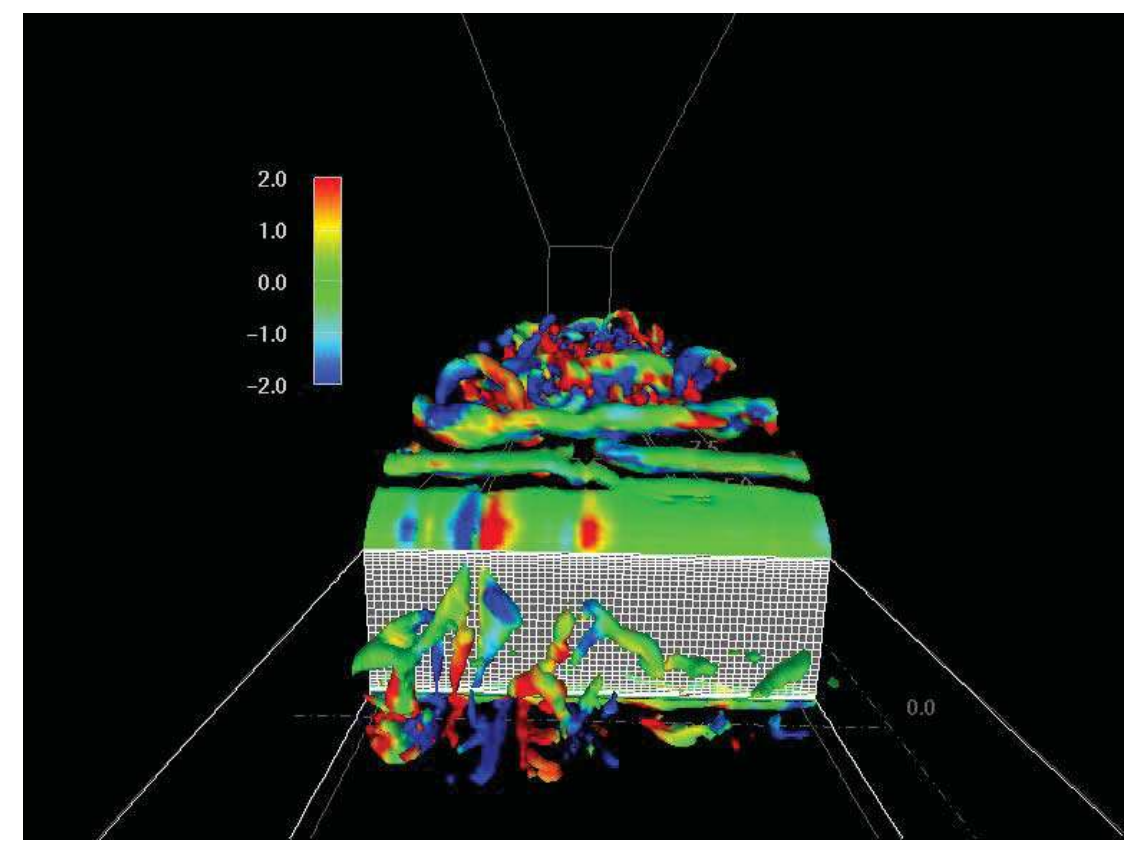

Figure 21. Instantaneous view of figure 20 animation.

spectra are just proportional to the spectrum of the aerodynamic noise emitted by the body. One sees that these pressure spectra start growing within the recirculation pocket above the obstacle (positions 25,25), together with the formation of big $\Lambda$ vortices. One sees in fact three marked peaks at point 25 , then two at point 26 and one dominant frequency at point 27 corresponding to the reattachment point of the recirculation bubble. At point 28 there is a strong attenuation of spectra. Further downstream (at the same elevation) spectra are quickly 


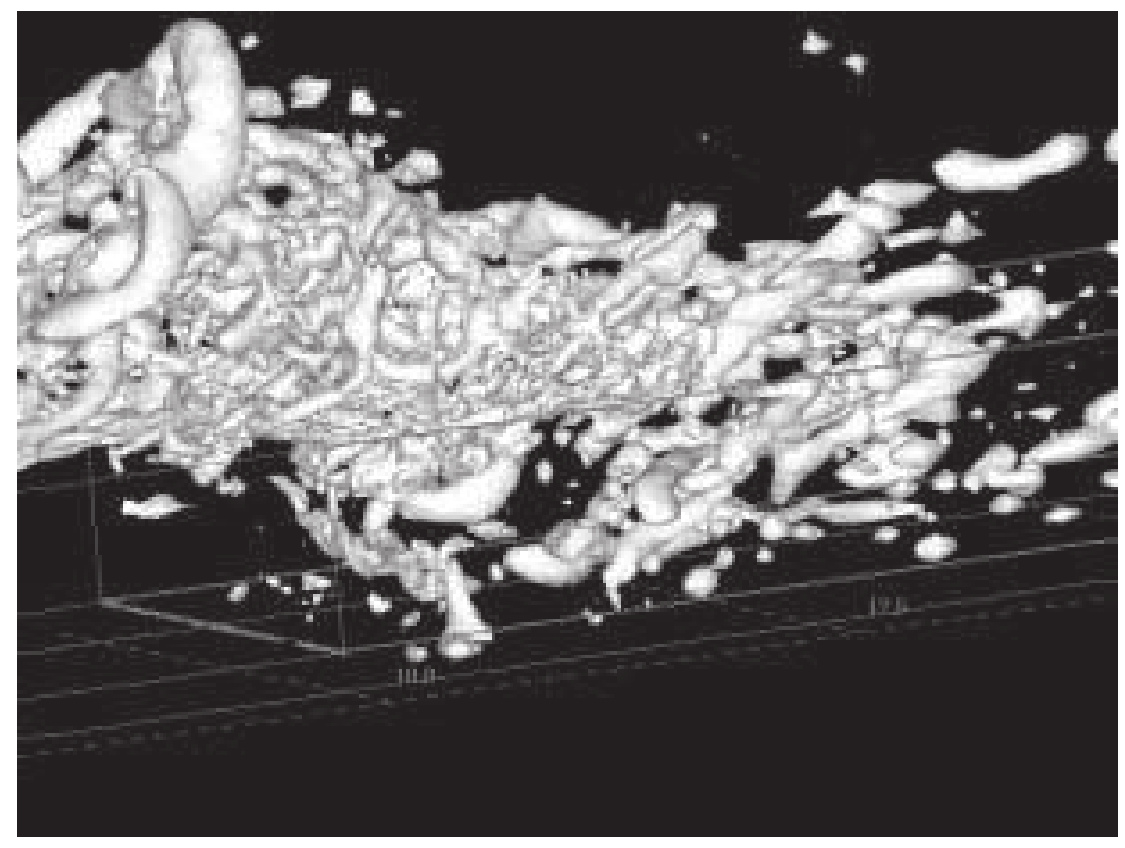

Figure 22. Animation of zoom of $Q$ behind the back-step.

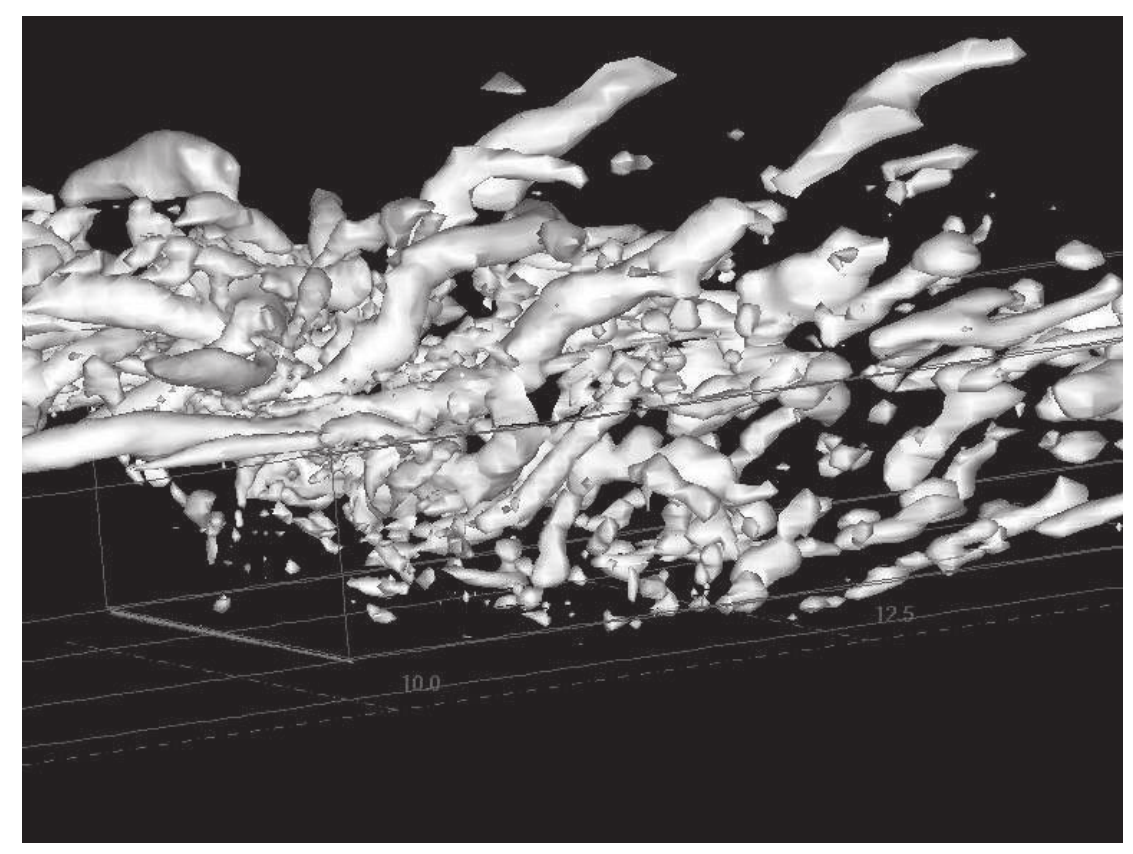

Figure 23. Instantaneous view of the animation of figure 22 .

damped. Closer to the bottom wall, spectra are important at points 45 and 40 (just behind the back-step), where they are much more monochromatic, then decay rapidly downstream. This shows without ambiguity that the noise emitted is associated with the presence of coherent vortices in the immediate neighbourhood of the wall. Velocity spectra are not shown here, but they are fairly well correlated with pressure spectra. 


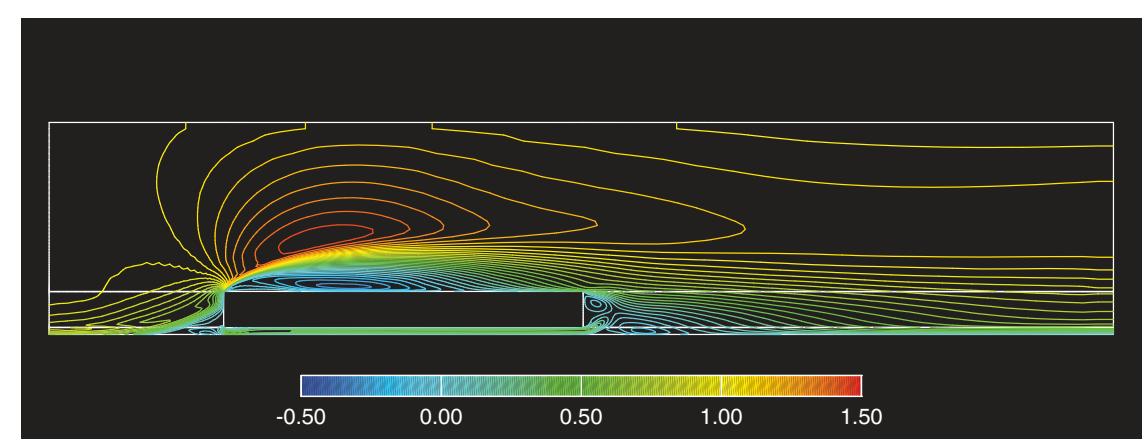

Figure 24. Mean longitudinal velocity.

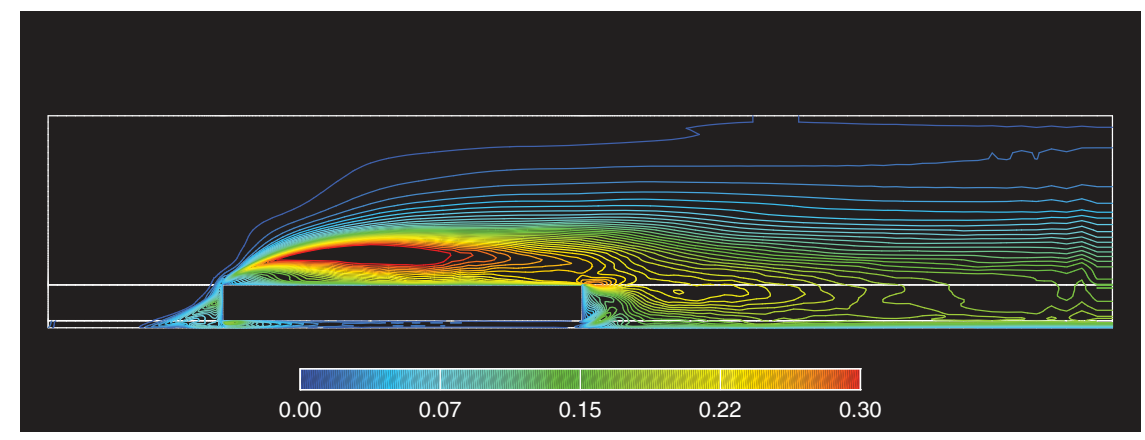

Figure 25. Rms longitudinal velocity.

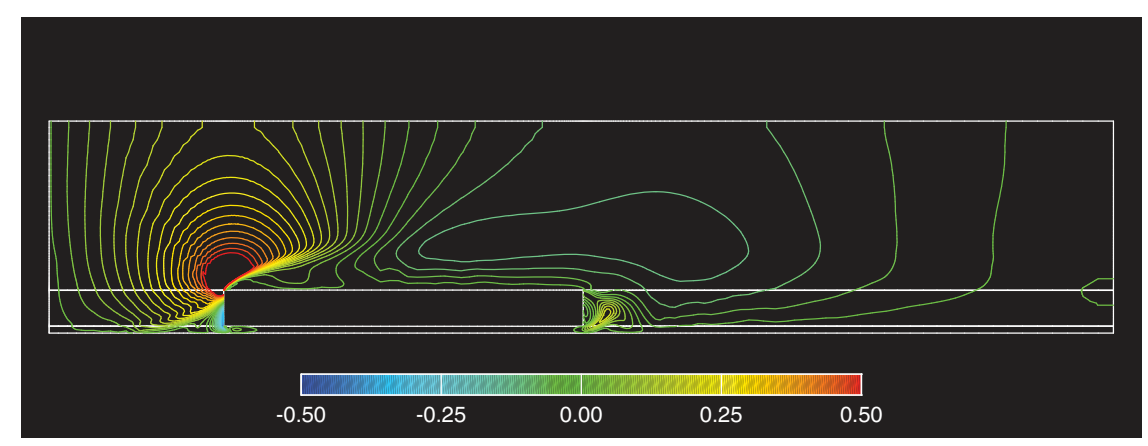

Figure 26. Mean vertical velocity.

\section{Conclusion and perspectives}

While biologists decipher the structure of the human genome, decisive progresses concerning the structure of turbulence and of vortices it contains have been made thanks to deterministic numerical solutions of the Navier-Stokes equation. Simple and effective algorithms allow us to visualize coherent vortices and follow their evolution in time. In this respect, the $Q$ criterion turns out to be a robust and efficient vortex analysis tool, together with the vorticity vector modulus. A considerable advance has been made in the last ten years with the recourse to LES, where small-scale fluctuations are filtered out and modelled with the aid of intelligent dynamic eddy viscosities and diffusivities: 'intelligent' and 'dynamic' in the sense that these eddy coefficients automatically cancel out in regions of space where the flow is laminar or transitional. Indeed, an extra eddy viscosity may very well inhibit the development of unstable waves, in the vicinity 


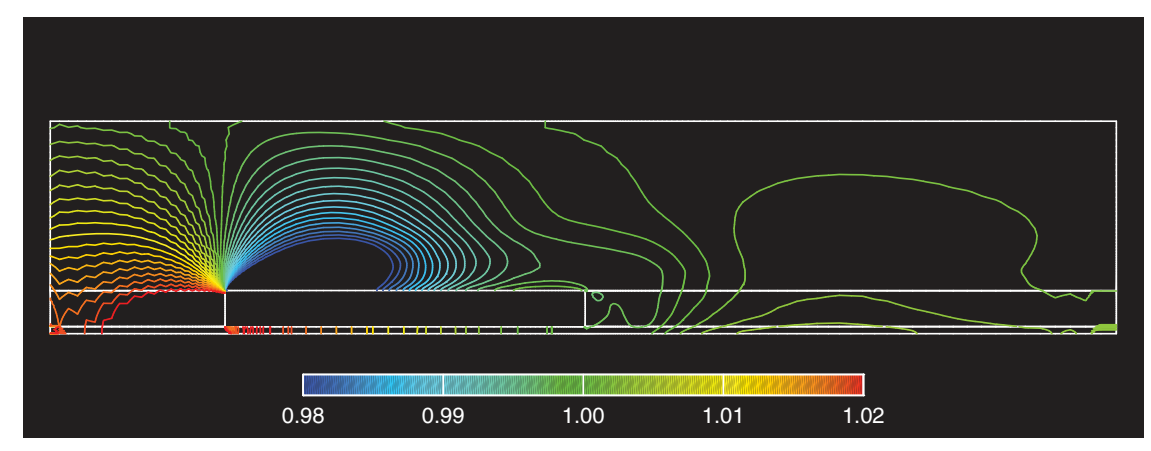

Figure 27. Mean pressure.

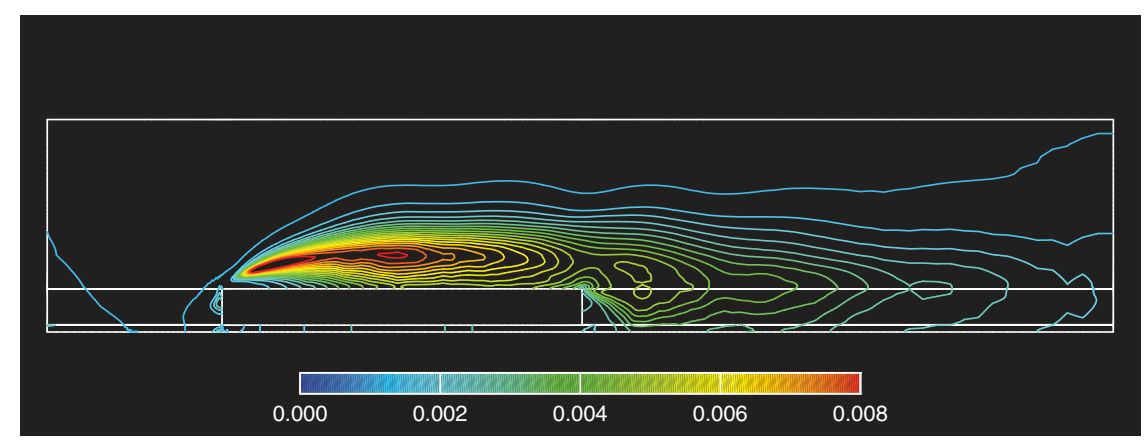

Figure 28. Rms pressure.

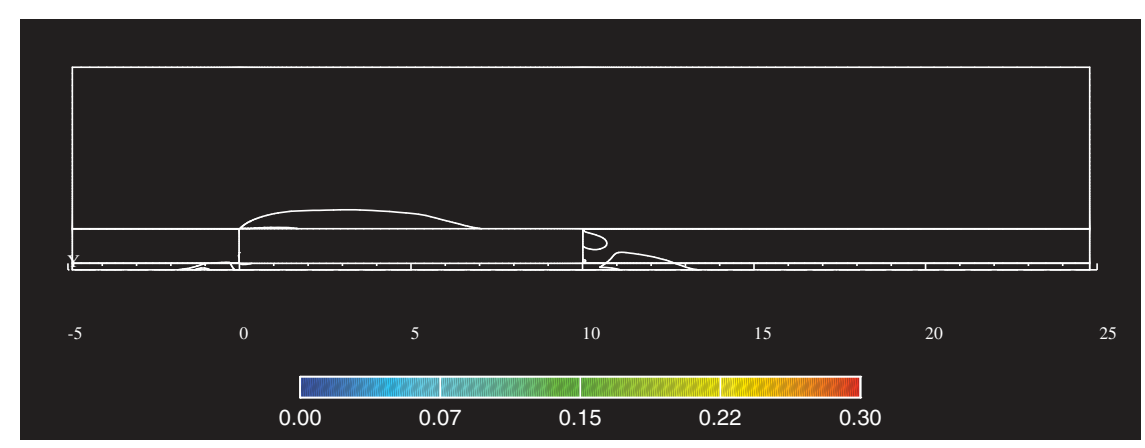

Figure 29. Zero-mean longitudinal velocity map.

of walls for instance, resulting in an unwanted relaminarization. Well carried LES of the type presented above are now well known to predict correctly basic first- and second-order statistics of free or wall-bounded turbulent shear flows.

In the present paper, we have attended to addressing the capability of LES to predict properly coherent-vortex dynamics. This has been done on the basis of a review of recent Grenoble works involving constant-density isotropic or weakly compressible shear flows (either free or wall bounded).

In isotropic decaying turbulence, we have recovered thanks to LES the thin longitudinal vortices found before by other authors using DNS. However, we have not found evidence that these vortices are formed initially by vortex-sheet roll-up. The way vortices form seems to depend upon initial conditions. 


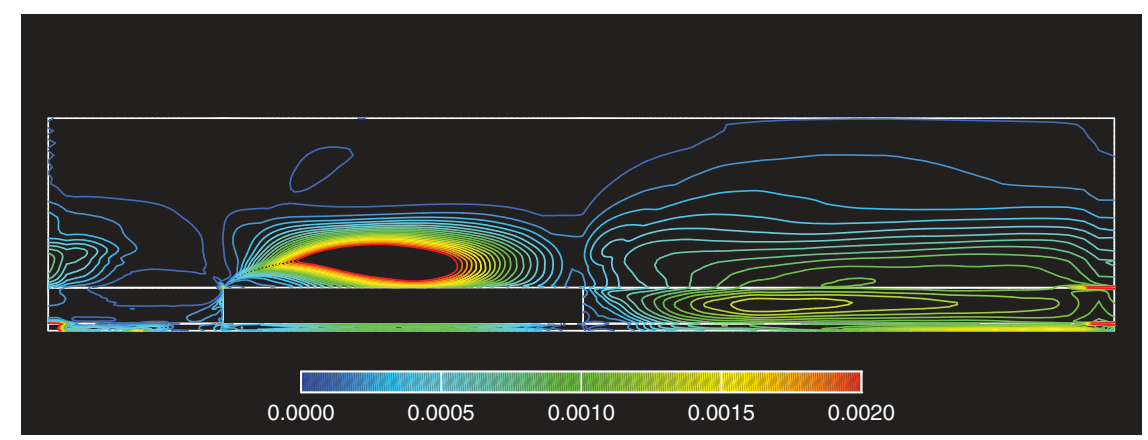

Figure 30. Mean eddy viscosity.

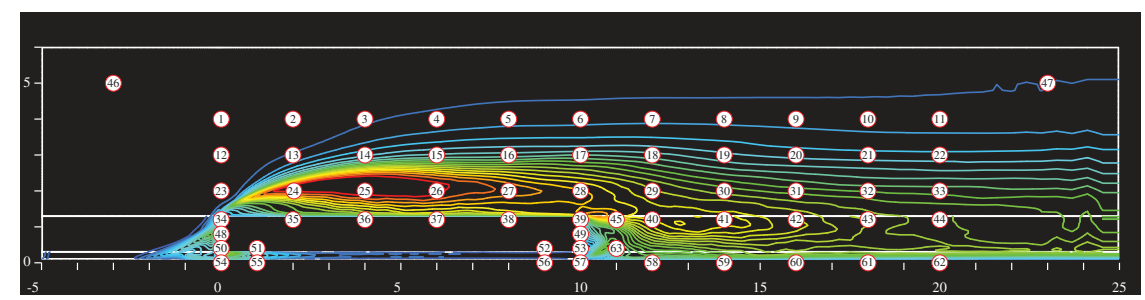

Figure 31. Location of the numerical probes.

In the very wide class of turbulent shear flows considered with LES, we find that coherent vortices are, roughly speaking, of two types: spiral Kelvin-Helmholtz vortices, and longitudinal vortices. Kelvin-Helmholtz vortices are of course the result of Kelvin-Helmholtz instability, and well documented in laboratory experiments and DNS. Big longitudinal vortices may form as the result of a longitudinal deformation of spanwise Kelvin-Helmholtz vortices in separated flows. The origin of thin quasi-longitudinal vortices forming in turbulent boundary layers is less clear, but again they have been recovered in experiments and DNS. The fact that we find these vortices in LES is a good validation for these methods to capture vortex dynamics.

Vortex control by manipulation of these vortices either at the level of their generation or during their life is essential in order to reduce drag and noise in aero- and hydrodynamics. This is linked in particular to the control of separation and/or associated recirculation, in order in particular to reduce the drag. Other examples of control are the reduction of the excessive noise caused by a plane turbojet engine during takeoff, or aeroacoustic noise of an external rear-view mirror on a passenger car. Thanks to the control of turbulence in all situations where it occurs (transport, engines and combustion chambers, nuclear engineering), one may expect a reduction of $20-30 \%$ of world global energetic consumption. An important application of turbulence control is also the reduction of atmospheric, oceanic, river and lake pollution. All these studies should associate fine non-stationary numerical simulations of the LES type with more industrial models of the RANS type. In this respect, the approach based on the so-called non-stationary RANS methods should be viewed more like a loosely resolved LES than like an non-stationary solution of the Reynolds equation. This is obvious in the case of all statistically stationary turbulent flows (such as a wake, mixing layer, back-step etc), where the Reynolds equation cannot have any time dependence. It is well known that, when a clear shedding frequency of vortices may be identified in the flow, phase averaging with respect to this signal gives rise to a non-stationary equation similar to the Reynolds equation. But phase averaging is not a well defined operator downstream of shear flows, where coherent vortices become unpredictable. It cannot be defined at all in isotropic turbulence if no solution of the flow is known. 


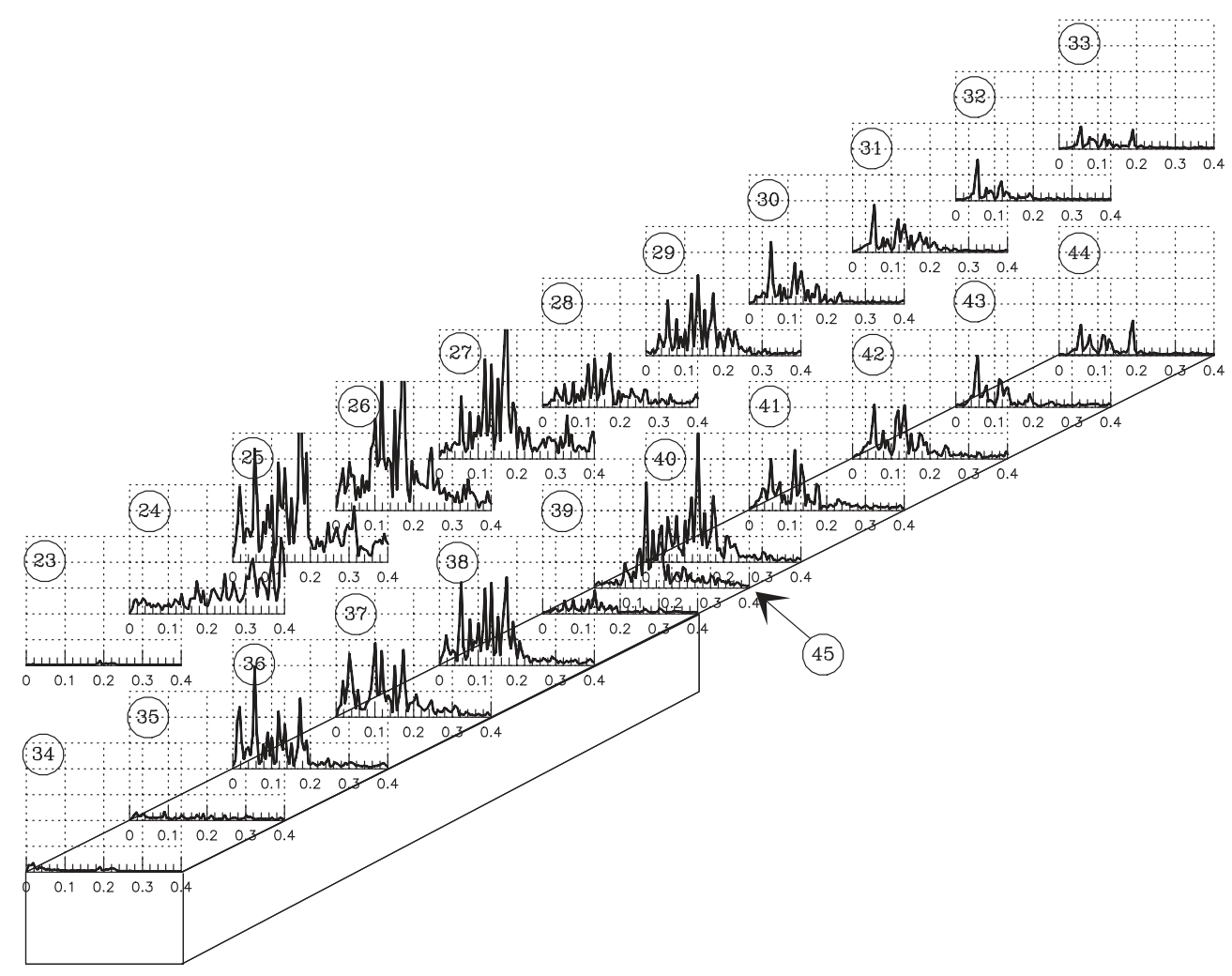

Figure 32. Pressure spectra at various locations.

\section{Acknowledgments}

We thank ONERA (Toulouse) for providing us with a PSE code allowing us to define upstream conditions in the boundary-layer simulation. Part of the simulations was carried out on the NEC SX5 of CNRS Institut du Développement des Ressources en Informatique Scientifique. The back-step and obstacle simulations were supported respectively by Peugeot-Citroën (PSA) and by the consortium ARPEGES (PSA-ALSTOM-SNCF).

\section{References}

[1] Siggia E D 1981 Numerical study of small-scale intermittency in three-dimensional turbulence J. Fluid Mech. $107375-406$

[2] She Z S, Jackson E and Orszag S A 1990 Nature 344226

[3] Vincent A and Ménéguzzi M 1991 The spatial structure and statistical properties of homogeneous turbulence J. Fluid Mech. 225 1-20

[4] Jimenez J and Wray A A 1998 On the characteristics of vortex filaments in isotropic turbulence J. Fluid Mech. 373 255-85

[5] Métais O and Lesieur M 1992 Spectral large-eddy simulations of isotropic and stably-stratified turbulence J. Fluid Mech. 239 157-94

[6] Lesieur M 1997 Turbulence in Fluids 3rd edn (Dordrecht: Kluwer) pp 1-515

[7] Cadot O, Douady S and Couder Y 1995 Characterization of very low pressure events in 3D turbulence Phys. Fluids 7630 .

[8] Robinson S 1991 Coherent motions in the turbulent boundary layer Annu. Rev. Fluid Mech. 23 601-39

[9] Dubief Y and Delcayre F 2000 On coherent-vortex identification in turbulence J. Turbulence 1011

[10] Ossia S and Lesieur M 2000 Energy backscatter in large-eddy simulations of three-dimensional incompressible isotropic turbulence J. Turbulence 1010 
[11] Lamballais E, Métais O and Lesieur M 1998 Spectral-dynamic model for large-eddy simulations of turbulent rotating channel flow Theor. Comput. Fluid Dyn. 12 149-77

[12] André J C and Lesieur M 1977 Influence of helicity on high Reynolds number isotropic turbulence J. Fluid Mech. 81 187-207

[13] Lesieur M and Ossia S 2000 3D isotropic turbulence at very high Reynolds numbers: EDQNM study J. Turbulence 1007

[14] Ackermann C and Métais O 2001 A modified selective structure function subgrid-scale model J. Turbulence 2011

[15] Ducros F, Comte P and Lesieur M 1996 Large eddy simulation of transition to turbulence in a boundary layer developing over a flat plate J. Fluid Mech. 326 1-36

[16] Herbert T 1997 Parabolized stability equations Annu. Rev. Fluid Mech. 29 487-526

[17] Airiau C 1994 Stabilité linéaire et faiblement non linéaire d'une couche limite laminaire incompressible par un système d'équations parabolisé (PSE) PhD Toulouse

[18] Christensen K and Adrian R 2002 The velocity and acceleration signatures of small-scale vortices in turbulent channel flow J. Turbulence $\mathbf{3} 023$

[19] Spalart P 1988 Direct simulation of a turbulent boundary layer up to $R_{\theta}=1410$ J. Fluid Mech. 187 61-98

[20] Danet A 2001 Influence des conditions amont sur l'écoulement derrière une marche par la simulation des grandes échelles $P h D$ Grenoble

[21] Le H, Moin P and Kim J 1997 Direct numerical simulation of turbulent flow over a backward-facing step J. Fluid Mech. 330 349-74

[22] Lund T, Wu X and Squires KD 1998 Generation of turbulent inflow data for spatially-developing boundarylayer simulations J. Comput. Phys. 140 233-58

[23] Schlichting H 1979 Boundary-Layer Theory (New York: McGraw-Hill)

[24] Rockwell D and Kniselly C 1980 Observation of the 3-dimensional nature of unstable flow past a cavity Phys. Fluids 23 239-40

[25] Mas D 2000 Rayonnement acoustique d'une cavité rectangulaire soumise à un écoulement turbulent $P h D$ Grenoble

[26] Kiya M and Sasaki S 1983 Structure of a turbulent separation bubble J. Fluid Mech. 137 83-113 